\title{
Gravitational waves from a very strong electroweak phase transition
}

\author{
Leonardo Leitao* and Ariel Mégevand ${ }^{\dagger}$ \\ IFIMAR (UNMdP-CONICET) \\ Departamento de Física, Facultad de Ciencias Exactas y Naturales, \\ UNMdP, Deán Funes 3350, (7600) Mar del Plata, Argentina
}

\begin{abstract}
We investigate the production of a stochastic background of gravitational waves in the electroweak phase transition. We consider extensions of the Standard Model which can give very strongly first-order phase transitions, such that the transition fronts either propagate as detonations or run away. To compute the bubble wall velocity, we estimate the friction with the plasma and take into account the hydrodynamics. We track the development of the phase transition up to the percolation time, and we calculate the gravitational wave spectrum generated by bubble collisions, magnetohydrodynamic turbulence, and sound waves. For the kinds of models we consider, we find parameter regions for which the gravitational waves are potentially observable at the planned space-based interferometer eLISA. In such cases, the signal from sound waves is generally dominant, while that from bubble collisions is the least significant of them. Since the sound waves and turbulence mechanisms are diminished for runaway walls, the models with the best prospects of detection at eLISA are those which do not have such solutions. In particular, we find that heavy extra bosons provide stronger gravitational wave signals than tree-level terms.
\end{abstract}

\section{Introduction}

In a cosmological first-order phase transition a stochastic background of gravitational waves (GWs) is generated, which could be observed in proposed gravitational wave detectors in space [1, 2, 3, 4, 5]. In particular, gravitational radiation which was formed at the scale of the electroweak phase transition (i.e., at a temperature $T \sim 100 \mathrm{GeV}$ ) has a characteristic frequency which, after redshifted, would be around the order of the $\mathrm{mHz}$ today. This is close to the sensitivity range of the observatory eLISA [5], which is scheduled for launch in 2034. This laser interferometer has a peak frequency in the range of $10^{-3}-10^{-2} \mathrm{~Hz}$.

*E-mail address: lleitao@mdp.edu.ar

${ }^{\dagger}$ Member of CONICET, Argentina. E-mail address: megevand@mdp.edu.ar 
Although in the minimal Standard Model (SM) the electroweak phase transition is not of first order, there are several extensions of the SM for which this transition is strongly first-order. The strength of the phase transition can be characterized by the value of the Higgs field in the broken-symmetry phase, $\phi_{b}$. Thus, a phase transition is usually said to be strongly first-order if this value fulfils the condition $\phi_{b} / T>1$, which is required for a successful electroweak baryogenesis [6]. However, for the generation of gravitational waves this condition may be not enough. A first order phase transition occurs via the nucleation and expansion of bubbles. The collisions of bubble walls and the motions caused in the plasma produce gravitational waves. For the GW signal to be sizeable, the bubble wall velocity as well as the energy which is transferred to fluid motions must be large enough. This generally requires significant supercooling and large latent heat. Thus, in principle, the stronger the phase transition, the larger the amplitude of the generated GWs.

The phase transition provides three sources of gravitational waves, namely, bubble collisions [7, 8, 9, 10, 11], magnetohydrodynamic (mhd) turbulence [12, 13, 14, 15, 16, 17], and sound waves [18]. The corresponding GW signals have been calculated as functions of a few relevant quantities which can be computed from the dynamics of the phase transition. These are the temperature $T_{*}$, the bubble wall velocity $v_{w}$, the duration of the phase transition $\beta^{-1}$, the ratio of the released energy to the energy in radiation, $\alpha$, and the efficiency factors $\kappa$. The latter give the fraction of the available energy which fuels a particular generating mechanism, and have been determined as a function of other parameters such as $\alpha$ and $v_{w}$ [12, 19, 20, 21, 22]. These results have allowed for several model-independent studies [23, 24, 25, 26, 27], in which the GW spectrum was computed as a function of the parameters $\alpha, \beta, v_{w}$, and $T_{*}$.

Specific models have also been considered, for which the gravitational radiation was computed as a function of model parameters [27, 28, 29, 30, 31, 32, 33]. Such a parameter variation is certainly more realistic, since the quantities $\alpha, \beta, v_{w}, T_{*}$ are not independent. However, these investigations rely on several approximations.

In particular, a key quantity for the calculation is the wall velocity. Unfortunately, computing the wall velocity is a very difficult task, which involves microphysics [34] and hydrodynamics [35, 36, 37]. Complete calculations of $v_{w}$ only exist in the non-relativistic approximation [38, 39, 40] and in the ultra-relativistic limit [41] (see also [42]). Due to these difficulties, the wall velocity is often set by hand in GW computations. For instance, it is very common to assume a Jouguet detonation [28, 29, 30, 31, 32], although this is generally incorrect [43]. Alternatively, one may choose a "representative" value for a detonation wall (e.g., $v_{w}=0.95$ [27]). However, the detonation velocity may vary between the speed of sound $\left(c_{s} \approx 0.58\right)$ and the speed of light [35, 36, 37. Another way of avoiding the calculation of the wall velocity is to consider runaway walls (i.e., $v_{w} \simeq 1$ ) [27, 33]. The condition for a wall to run away in a given model is relatively easy to determine. However, there are many models which do not allow for runway walls.

On the other hand, the quantities $\alpha, \beta$, and $v_{w}$ depend on the temperature. It is usual to compute these quantities at the nucleation temperature $T_{n}$ (and to assume $T_{*} \simeq T_{n}$ ), which is defined as that at which there is one bubble in a Hubble volume. However, it is well known that this temperature $T_{n}$ corresponds to the onset of nucleation. The supercooling continues during the development of the phase transition, and percolation takes place at a smaller temperature $T_{p}$ (the value $T_{*}$ actually corresponds to the reheat 
temperature after percolation). Since GW generation begins once bubbles begin to collide, this additional supercooling should be taken into account, especially for the case of very strong phase transitions.

In this paper, we consider physical variations of model parameters for concrete extensions of the SM. Our aim is to study very strong electroweak phase transitions which allow for detonations or runaway walls. The main difference of our approach with previous works is that we attempt a realistic computation of the phase transition dynamics. For each model we estimate the wall velocity and we follow the nucleation and growth of bubbles until percolation. We performed a similar analysis in our earlier work [44]. Here we extend that analysis by considering models with stronger phase transitions, and we improve the calculation by taking into account the saturation of the friction force at high velocities (which in particular is responsible for the appearance of runaway solutions). For that aim we use an approximation for the friction developed in Refs. [45, 22], which allows us to treat both detonations and runaway walls.

The paper is organized as follows. In the next section we describe our treatment of the dynamics of the phase transition. In Sec. 3 we write down the formulas for the GW signal produced by the different mechanisms, and we discuss the computation of the relevant parameters from the phase transition dynamics. In Sec. 4 we present our numerical results. We discuss on the dynamics of such very strong electroweak phase transitions and the consequences for the generation of gravitational waves. In Sec. 5 we summarize our conclusions.

\section{The electroweak phase transition}

\subsection{The free energy}

It is well known that in the SM the electroweak phase transition is a smooth crossover [46], and one needs to go beyond this model to have a first-order phase transition. For the SM, the tree-level potential is of the form

$$
V_{0}(\phi)=-m^{2} \phi^{2}+\frac{\lambda}{4} \phi^{4}+\frac{\lambda}{4} v^{4}
$$

where $\phi$ is the background Higgs field, defined by $\left\langle H^{0}\right\rangle \equiv \phi / \sqrt{2}$. The parameters in Eq. (11) are related to the vacuum expectation value and the mass of the Higgs boson by $v=\sqrt{2 / \lambda} m=246 \mathrm{GeV}, m_{H}=\sqrt{2 \lambda v^{2}}=125 \mathrm{GeV}$, and we have chosen the constant term so that the true-vacuum energy density $V_{0}(v)$ vanishes. The zero-temperature effective potential is given, to one-loop order, by

$$
V(\phi)=V_{0}(\phi)+V_{1}(\phi)
$$

where the renormalized zero-temperature correction is given by

$$
V_{1}(\phi)=\sum_{i} \frac{ \pm g_{i}}{64 \pi^{2}}\left[m_{i}^{4}(\phi)\left(\log \frac{m_{i}^{2}(\phi)}{m_{i}^{2}(v)}-\frac{3}{2}\right)+2 m_{i}^{2}(\phi) m_{i}^{2}(v)-\frac{m_{i}^{4}(v)}{2}\right] .
$$


Here, the sum is over all particle species which couple to the Higgs, $g_{i}$ is the number of degrees of freedom (d.o.f.) of particle species $i$, the upper sign correspond to bosons, the lower sign to fermions, and $m_{i}$ are the Higgs-dependent particle masses. In this expression, we have chosen renormalization conditions that the tree-level values of the minimum, the Higgs mass, and the true vacuum energy density, are not shifted by radiative corrections, i.e., $V_{1}(v)=V_{1}^{\prime}(v)=V_{1}^{\prime \prime}(v)=0$ (a prime indicates a derivative with respect to $\phi$ ). The relevant species are those with larger couplings to the Higgs. For the SM, these are the $Z$ and $W$ bosons, the top quark, and the Higgs and Goldstone bosons. For simplicity we shall ignore the Higgs sector in the one-loop radiative corrections.

The dynamics of the phase transition is determined by the free energy density (also called finite-temperature effective potential). To one-loop order, it is given by

$$
\mathcal{F}(\phi, T)=V(\phi)+\mathcal{F}_{1}(\phi, T)
$$

where the finite-temperature corrections are given by [47]

$$
\mathcal{F}_{1}(\phi, T)=\sum_{i}\left( \pm g_{i}\right) T \int \frac{d^{3} p}{(2 \pi)^{3}} \log \left(1 \mp e^{-E_{i} / T}\right)
$$

with $E_{i}=\sqrt{p^{2}+m_{i}^{2}(\phi)}$. As is well known, for $m_{i} / T \ll 1$ we have the expansion

$$
\mathcal{F}_{1}(\phi, T)=-\sum_{i} \frac{g_{i} c_{i}^{\prime} \pi^{2} T^{4}}{90}+\sum_{i} \frac{g_{i} c_{i} T^{2} m_{i}^{2}(\phi)}{24}-\sum_{\text {bosons }} \frac{g_{i} T m_{i}^{3}(\phi)}{12 \pi}+\mathcal{O}\left(m^{4}\right),
$$

where $c_{i}=1(1 / 2)$ and $c_{i}^{\prime}=1(7 / 8)$ for bosons (fermions). For the particles of the SM, the masses are of the form

$$
m_{i}(\phi)=h_{i} \phi
$$

and we shall assume for simplicity this dependence for the extra species as well.

Bosons with masses of the form (7) give cubic terms proportional to $-h_{i}^{3} T \phi^{3}$ in Eq. (6)), which can make the phase transition strongly first-order for large $h_{i}$. The contributions from the SM gauge bosons are not enough to cause a strongly first-order phase transition. For that reason, it is usual to consider SM extensions with extra bosons (such as e.g. the MSSM). However, when the resummed daisy diagrams are included, it turns out that the extra-particle contributions to the thermal cubic term are suppressed, except for the transverse polarizations of gauge bosons 1 . To take into account this suppression, we will just subtract the corresponding cubic terms. For the models we will consider, the strength of the phase transition does not rely on such thermally-induced cubic terms. We are interested in cases in which the order parameter $\phi / T$ is not necessarily small enough for the validity of the approximation ([6). Therefore, we will compute the complete oneloop correction (5), but we shall add a term $\sum g_{i} T m_{i}^{3} /(12 \pi)$ (where the sum includes all the bosonic d.o.f., except for the transverse polarizations of gauge bosons) to take into account the suppression of the cubic terms.

For the effective potential (44), the symmetric value $\phi=0$ is the absolute minimum at high temperatures. In general, there is a range of temperatures in which this minimum

\footnotetext{
${ }^{1}$ For a model in which the gauge fields play a relevant role, it should be taken into account the fact that the finite-temperature effective potential is not gauge-invariant [48.
} 
coexists with a symmetry-breaking minimum $\phi_{m}(T)$. At low temperatures, $\phi_{m}$ is the absolute minimum, which takes the value $\phi_{m}=v$ at zero temperature. The two minima are degenerate at the critical temperature $T_{c}$, i.e., $\mathcal{F}\left(0, T_{c}\right)=\mathcal{F}\left(\phi_{m}, T_{c}\right)$. The unbrokenand broken-symmetry phases are characterized by the free energy densities $\mathcal{F}_{u}(T)=$ $\mathcal{F}(0, T)$ and $\mathcal{F}_{b}(T)=\mathcal{F}\left(\phi_{m}(T), T\right)$, respectively. The pressure in each phase is given by $p=-\mathcal{F}$, the energy density is given by $\rho=\mathcal{F}-T d \mathcal{F} / d T$, and the enthalpy density by $w=\rho+p=-T d \mathcal{F} / d T$. At the critical temperature, the pressures are equal, $p_{u}\left(T_{c}\right)=$ $p_{b}\left(T_{c}\right)$, but the energy density is different and the latent heat $L$ is defined by the difference $L=\rho_{u}\left(T_{c}\right)-\rho_{b}\left(T_{c}\right)$.

\subsection{Phase transition dynamics}

Below the critical temperature, spherical bubbles of the broken-symmetry phase nucleate with a rate [49]

$$
\Gamma(T) \simeq A(T) e^{-S_{3}(T) / T},
$$

with $A(T)=\left[S_{3}(T) /(2 \pi T)\right]^{3 / 2} T^{4}$, where $S_{3}$ is the three-dimensional instanton action

$$
S_{3}=4 \pi \int_{0}^{\infty} r^{2} d r\left[\frac{1}{2}\left(\frac{d \phi}{d r}\right)^{2}+V_{T}(\phi(r))\right],
$$

and

$$
V_{T}(\phi) \equiv \mathcal{F}(\phi, T)-\mathcal{F}(0, T) .
$$

The configuration of the nucleated bubble is a solution of the equations

$$
\frac{d^{2} \phi}{d r^{2}}+\frac{2}{r} \frac{d \phi}{d r}=\frac{d V_{T}}{d \phi}, \quad \frac{d \phi}{d r}(0)=0, \quad \lim _{r \rightarrow \infty} \phi(r)=0 .
$$

The function $S_{3}(T)$ diverges at $T=T_{c}$ and, hence, we have $\Gamma\left(T_{c}\right)=0$. As $T$ decreases below $T_{c}, S_{3}$ decreases and $\Gamma$ grows.

The temperature variation is governed by the equation $d T / d t=-H T$, and the expansion rate is given by the Friedmann equation, $H=\sqrt{8 \pi G \rho / 3}$, where $G$ is Newton's constant. At the beginning of the phase transition the energy density is given by $\rho=\rho_{u}(T)$, and we have a simple equation for $T(t)$. On the other hand, when bubbles of the brokensymmetry phase are already nucleated, we have regions with different equations of state. If the temperature $T$ were homogeneous, we would have $\rho_{b}(T)<\rho_{u}(T)$. However, the energy difference between the two phases goes into reheating of the plasma as well as bulk fluid motions. For the fast phase-transition fronts we are interested in (namely, detonations or runaway walls), the released energy is distributed behind the walls, i.e., inside the bubbles. Therefore, the temperature $T_{u}$ outside the bubbles is homogeneous. Since bubbles nucleate in the symmetric phase, the rate $\Gamma$ depends only on this temperature. In spite of this, in the Friedmann equation we have to take into account also the energy density in the broken-symmetry phase. Nevertheless, energy conservation insures that the average energy density remains essentially unchanged, i.e., the average energy density $\bar{\rho}_{b}$ inside the bubbles must be essentially the same as outside, $\bar{\rho}_{b}=\rho_{u}\left(T_{u}\right)$. Thus, we have

$$
\frac{d T_{u}}{d t}=-\sqrt{\frac{8 \pi G \rho_{u}\left(T_{u}\right)}{3}} T_{u}
$$


From Eq. (12) we readily obtain $T_{u}(t)$ and, thus, $\Gamma(t)=\Gamma\left(T_{u}(t)\right)$.

Let us call $t_{c}$ the time at which the Universe reaches the critical temperature. Then, at time $t>t_{c}$, the development of the phase transition is characterized by the fraction of volume $f_{u}(t)$ in the unbroken-symmetry phase or, equivalently, that in the brokensymmetry phase, $f_{b}=1-f_{u}$. Taking into account bubble overlapping and the fact that bubbles can only nucleate in the symmetric phase 2 , we have [50]

$$
f_{u}(t)=\exp \left[-\frac{4 \pi}{3} \int_{t_{c}}^{t} d t^{\prime} \Gamma\left(T_{u}^{\prime}\right)\left(\frac{a^{\prime}}{a}\right)^{3} R_{b}\left(t^{\prime}, t\right)^{3}\right]
$$

where we use the notation $T_{u}^{\prime}=T_{u}\left(t^{\prime}\right), a^{\prime}=a\left(t^{\prime}\right), a$ is the scale factor (which is determined by the equation $\dot{a} / a=H$ ), and $R_{b}$ is the radius of a bubble which nucleated at time $t^{\prime}$ and expanded until time $t$.

The initial bubble radius can be obtained from the bubble configuration at the nucleation time $t^{\prime}$. Nevertheless, it is well known that for a phase transition at the electroweak scale this initial radius is negligible in comparison with the final bubble size. The bubble wall velocity at a given time $t^{\prime \prime}$ between $t^{\prime}$ and $t$ is a function of the temperature outside the bubble, $v_{w}^{\prime \prime}=v_{w}\left(T_{u}^{\prime \prime}\right)$ (see below). Thus, the bubble radius at time $t$ is given by

$$
R_{b}\left(t^{\prime}, t\right)=\int_{t^{\prime}}^{t} v_{w}^{\prime \prime} \frac{a}{a^{\prime \prime}} d t^{\prime \prime}
$$

where $a^{\prime \prime}=a\left(t^{\prime \prime}\right)$.

\subsection{The wall velocity}

The equation of motion for the wall can be obtained from the field equation (see e.g., [38]). For a wall propagating towards the positive $z$ direction with velocity $v_{w}(t)$ and Lorentz factor $\gamma_{w}=1 / \sqrt{1-v_{u w}^{2}}$, we may assume a field profile of the form $\phi(z, t)=\phi_{0}\left(\left(z-z_{w}\right) \gamma_{w}\right)$, with $\phi_{0}(z)$ a function 3 which varies between the minima $\phi=\phi_{m}$ and $\phi=0$ in a small range (the wall width) around the wall position $z_{w}(t)[22$. Then, the wall equation can be written as

$$
\sigma_{0} \gamma_{w}^{3} \dot{v}_{w}=\int \frac{\partial \mathcal{F}}{\partial \phi} \frac{\partial \phi}{\partial z} d z+\int d z \sum_{i} g_{i} \frac{d m_{i}^{2}}{d \phi} \frac{d \phi}{d z} \int \frac{d^{3} p}{(2 \pi)^{3} 2 E_{i}} \delta f_{i},
$$

where $\sigma_{0}=\int\left[\phi_{0}^{\prime}(z)\right]^{2} d z$ (notice that all the integrands vanish outside the wall, where $d \phi / d z=0)$, and $\delta f_{i}$ are the deviations from equilibrium of the particles distribution functions. The left-hand side of Eq. (15) is just the proper acceleration of the wall times its surface energy density at rest. Hence, the right hand side is the net force $F_{\text {net }}$ (per unit area) acting on the wall, and we may write $F_{\text {net }}=F_{\mathrm{dr}}+F_{\mathrm{fr}}$, where the driving force $F_{\mathrm{dr}}$ is given by the first term in Eq. (15) and the friction force $F_{\text {fr }}$ is given by the last term.

\footnotetext{
${ }^{2}$ The calculation is more involved for deflagration bubbles, which are preceded by shock fronts which reheat the plasma. Thus, the nucleation rate is also suppressed in the reheated regions of the symmetric phase. For a treatment of this case, see [44].

${ }^{3} \mathrm{~A}$ specific tanh ansatz is often used.
} 
The driving force is relatively easy to calculate. Taking into account the fact that the temperature varies across the wall, this force can be written as (see e.g. [22]) $F_{\mathrm{dr}}=$ $\mathcal{F}_{u}-\mathcal{F}_{b}-\int\left(\partial \mathcal{F} / \partial T^{2}\right) d T^{2}$, which can be approximated by

$$
F_{\mathrm{dr}}=\mathcal{F}_{u}-\mathcal{F}_{b}-\left\langle\frac{\partial \mathcal{F}}{\partial T^{2}}\right\rangle\left(T_{u}^{2}-T_{b}^{2}\right),
$$

where $\langle Q\rangle$ means averaging the values of a given quantity $Q$ on each side of the wall, $\langle Q\rangle=\left(Q_{u}+Q_{b}\right) / 2$. For the cases of a detonation or a runaway wall, the temperature $T_{u}$ outside the bubble is given by Eq. (12), while the plasma inside the bubble is reheated. This reheating can affect significantly the driving force, which is sensitive to the departures of $T_{u}$ and $T_{b}$ from $T_{c}$.

The friction force is much more involved. To compute the deviations $\delta f_{i}$ it is necessary to consider a set of integro-differential Boltzmann equations for the particles with strongest couplings to the Higgs. The collision terms of the Boltzmann equations involve all the interactions of species $i$ with all other species. A detailed computation is very difficult, and has been attempted only for a few models [38, 39, 40]. Among other approximations, the deviations from equilibrium are assumed to be small in order to work to linear order in the perturbations. As a consequence, these calculations break down if the wall velocity is close to the speed of sound (where the hydrodynamics becomes very strong) as well as for ultra-relativistic wall velocities [42]. In the non-relativistic limit, the friction force is of the form [34] $F_{\mathrm{fr}}^{N R}=-\eta_{N R} v_{w}$. Analytic approximations for the friction coefficient $\eta_{N R}$ were derived in Refs. [51, 52]. For masses of the form (77) we havet,

$$
\eta_{N R}=\sum_{i} \frac{g_{i} h_{i}^{4}}{\Gamma} \int_{0}^{\phi_{c}} c_{1}(\phi)^{2} \phi^{2} \sqrt{2 V_{T_{c}}(\phi)} d \phi,
$$

where $\Gamma$ is an average interaction rate, $\phi_{c}=\phi_{m}\left(T_{c}\right)$, and

$$
c_{1}=\frac{1}{T_{u}^{2}} \int \frac{d^{3} p}{(2 \pi)^{3}} \frac{1}{E} \frac{e^{E_{i} / T_{u}}}{\left(e^{E_{i} / T_{u}} \mp 1\right)^{2}} .
$$

For the electroweak phase transition, the quantity $\Gamma$ is typically $\sim 10^{-2} T$, and can be chosen to fit the results of detailed numerical calculations. Most of the quantities appearing in Eq. (17) are not very sensitive to the temperature, and thus we evaluate them at $T=T_{c}$, which simplifies considerably the calculation. Nevertheless, we compute $c_{1}$ at $T=T_{u}$ since this factor is exponentially suppressed in the case of strong supercooling ( $c_{1}$ is often calculated to lowest order in $m / T$, but this approximation breaks down for $\left.T \ll T_{c}\right)$. The approximation $F_{\mathrm{fr}}^{N R}=-\eta_{N R} v_{w}$ assumes a fluid at rest, where $v_{w}$ is the wall velocity relative to this fluid. We actually have a fluid velocity $v$ which varies across the wall (see below). To take into account its effect, we may replace $v_{w}$ with the average $\left\langle v_{w}-v\right\rangle$. Thus, we have

$$
F_{\mathrm{fr}}^{N R}=-\eta_{N R}\left(v_{w}-\bar{v}\right)
$$

\footnotetext{
${ }^{4}$ The friction coefficient $\eta_{N R}$ receives also a contribution from infrared boson excitations [53]. However, this contribution is suppressed for strongly first-order phase transitions [51, 52, and we shall neglect it in this work.
} 
where $\bar{v}=\left(v_{b}+v_{u}\right) / 2$.

For an ultra-relativistic wall, the interactions are too slow in comparison to the passage of the wall, and it is not necessary to consider Boltzmann equations [41]. In this case, the complete distribution functions $f_{i}$ can be computed exactly, and the result is

$$
F_{\text {net }}^{U R}=V\left(\phi_{u}\right)-V\left(\phi_{b}\right)-\sum_{i} \frac{g_{i} c_{i} h_{i}^{2}}{24} T_{u}^{2} \phi_{b}^{2} .
$$

If this net force is positive, then the wall is accelerated. Actually, in the UR limit $\dot{v}_{w}$ vanishes due to the $\gamma_{w}$ factors in Eq. (15), which means that the wall velocity is almost constant, since it is very close to the limit $v_{w}=1$. Nevertheless, the gamma factor increases with time, as well as the kinetic and gradient energy of the Higgs field in the wall. On the other hand, if the UR force $F_{\text {net }}^{U R}$ is negative, then the wall will not reach the UR regime and, instead, will reach a terminal velocity $v_{w}<1$, with $F_{\text {net }}=0$.

To compute this terminal velocity, we must solve the equation

$$
F_{\mathrm{dr}}+F_{\mathrm{fr}}=0,
$$

where $F_{\mathrm{dr}}$ is given by Eq. (16) and $F_{\text {fr }}$ is a velocity dependent function. The expressions (19) and (20) assume either the NR or the UR limit. In order to take into account the possibility of intermediate cases, we shall use a phenomenological interpolation for the friction, which was introduced in Ref. [45. In the reference frame of the plasma in front of the wall we have 22

$$
F_{\mathrm{fr}}=-\frac{\eta_{N R} \eta_{U R} \gamma_{w} \bar{\gamma}\left(v_{w}-\bar{v}\right)}{\sqrt{\eta_{U R}^{2}+\eta_{N R}^{2} \gamma_{w}^{2} \bar{\gamma}^{2}\left(v_{w}-\bar{v}\right)^{2}}}
$$

where $\bar{\gamma}=1 / \sqrt{1-\bar{v}^{2}}$ and $\eta_{N R}, \eta_{U R}$ are free parameters which can be set to fit the values of the friction in the NR and the UR limits. Indeed, for small wall velocities Eq. (22) becomes $F_{\text {fr }}=-\eta_{N R}\left(v_{w}-\bar{v}\right)$ while for $v_{w} \rightarrow 1$ we have $F_{\text {fr }}=-\eta_{U R}$. Thus, $\eta_{N R}$ is the non-relativistic friction coefficient discussed above, while the parameter $\eta_{U R}$ is just given by the UR friction force. Notice that Eq. (20) does not give the UR friction force but the total force $F_{\text {net }}^{U R}$. The first two terms in Eq. (20) give the zero-temperature value of the force, while the last term comprises the hydrodynamics effects as well as the friction. For a given model, the UR friction can be obtained by subtracting from Eq. (20) the UR limit of the driving force (16). Thus, we have $\eta_{U R}=-\left(F_{\text {net }}^{U R}-F_{\mathrm{dr}}^{U R}\right)$. This coefficient will depend on the temperature and on parameters of the model.

\subsection{Hydrodynamics}

The forces acting on the wall depend on the fluid variables $T_{u}, T_{b}, v_{u}, v_{b}$. For detonations and runaway walls the fluid in front of the wall is unperturbed. Thus, $T_{u}$ is given by Eq. (12), $v_{u}$ vanishes5, and we only need to determine the values of $T_{b}$ and $v_{b}$. We are

\footnotetext{
${ }^{5}$ In the case of deflagrations the hydrodynamics is more involved, since a shock front propagates in front of the wall. In that case, the boundary conditions for the temperature and the fluid velocity must be imposed beyond the shock front. To determine $T_{u}$ and $v_{u}$ in front of the wall, the fluid equations in the symmetric phase must be solved.
} 
also interested in the complete temperature and velocity profiles behind the wall, in order to compute the energy injected in the plasma. Both the fluid equations inside the bubble and the relations between the variables on each side of the wall can be obtained from the conservation of the energy-momentum tensor. For hydrodynamics calculations it is usual to consider a simplification of the equation of state (EOS), namely, the bag EOS.

For the models we will consider, the particles are massless in the symmetric phase. Hence, in this phase the finite-temperature correction to the effective potential is just given by the first term in Eq. (6), and the free energy density is of the form

$$
\mathcal{F}_{u}=\varepsilon_{u}-a_{u} T_{u}^{4} / 3
$$

where $\varepsilon_{u}=V(0)$ is the false-vacuum energy density, and $a_{u}=\sum \pi^{2} g_{i} c_{i}^{\prime} / 30$ is the radiation constant. Thus, the pressure is given by $p_{u}=-\varepsilon_{u}+a_{u} T_{u}^{4} / 3$, the energy density is given by $\rho_{u}=\varepsilon_{u}+a_{u} T_{u}^{4}$, and the enthalpy density is given by $w_{u}=(4 / 3) a_{u} T_{u}^{4}$. The equation of state of the broken-symmetry phase is more involved. The bag approximation assumes that the free energy density is of the form of Eq. (23) also in this phase, i.e.,

$$
\mathcal{F}_{b}=\varepsilon_{b}-a_{b} T_{b}^{4} / 3
$$

Therefore, we have $p_{b}=-\varepsilon_{b}+a_{b} T_{b}^{4} / 3, \rho_{b}=\varepsilon_{b}+a_{b} T_{b}^{4}$, and $w_{b}=(4 / 3) a_{b} T_{b}^{4}$. For this model we have only vacuum and radiation components. The speed of sound in both phases is given by $c_{s}=1 / \sqrt{3}$. The critical temperature is determined by the relation $\Delta a T_{c}^{4}=3 \Delta \varepsilon$, where $\Delta \varepsilon=\varepsilon_{u}-\varepsilon_{b}$ and $\Delta a=a_{u}-a_{b}$, and the latent heat is given by $L=4 \Delta \varepsilon$.

For the bag EOS, the hydrodynamics depends essentially on two variables. These are the ratio of degrees of freedom, $a_{b} / a_{u}$, and the ratio of the released vacuum energy density to the radiation energy density,

$$
\alpha=\Delta \varepsilon /\left(a_{u} T_{u}^{4}\right) .
$$

Thus, for instance, the driving force (16) becomes

$$
F_{\mathrm{dr}}=\Delta \varepsilon\left(1-T_{b}^{2} T_{u}^{2} / T_{c}^{4}\right)
$$

and the temperatures $T_{b}$ and $T_{u}$ are related by $T_{b}^{4} / T_{u}^{4}=\left(a_{u} / a_{b}\right)\left(w_{b} / w_{u}\right)$. For a detonation, the ratio $w_{b} / w_{u}$, as well as the fluid velocity $v_{b}$, are functions of $\alpha$ and the wall velocity $v_{w}$. Analytic expressions can be found in Ref. [22]. For a runaway wall, instead, these quantities depend on $\alpha$ and the UR net force $F_{\text {net }}^{U R}$. We have [22]

$$
\frac{w_{b}}{w_{u}}=1+3(\alpha-\bar{F}), \quad v_{b}=\frac{3(\alpha-\bar{F})}{2+3(\alpha-\bar{F})},
$$

where

$$
\bar{F} \equiv F_{\text {net }}^{U R} / a_{u} T_{u}^{4}
$$

The limit $\bar{F}=0$ of Eqs. (27) matches the UR limit $v_{w}=1$ of the detonation result. For $\bar{F}>0$, the wall is accelerated and part of the energy and momentum accumulate in the wall. As a consequence, the reheating and fluid motions decrease. 
For a given model, we can calculate $\bar{F}$ from Eq. (20). Then, using Eqs. (26-27), we readily obtain the UR driving force and, hence, the friction parameter $\eta_{U R}$ [22],

$$
\frac{\eta_{U R}}{a_{u} T_{u}^{4}}=\alpha-\bar{F}-\frac{1}{3}\left(1-\frac{a_{b}}{a_{u}}\right) \sqrt{\frac{1+3(\alpha-\bar{F})}{a_{b} / a_{u}}} .
$$

Once this parameter is computed, the value of the wall velocity in the steady state regime can be obtained from the equation

$$
\Delta \varepsilon\left(1-T_{b}^{2} T_{u}^{2} / T_{c}^{4}\right)-\frac{\eta_{N R} \eta_{U R} \gamma_{w} \bar{\gamma}\left(v_{w}-\bar{v}\right)}{\sqrt{\eta_{U R}^{2}+\eta_{N R}^{2} \gamma_{w}^{2} \bar{\gamma}^{2}\left(v_{w}-\bar{v}\right)^{2}}}=0
$$

For a detonation, the fluid velocity in front of the wall vanishes and we have $\bar{v}=v_{b} / 2$.

The generation of gravitational waves depends on the kinetic energy of the wall as well as that in bulk fluid motions. For detonations or runaway walls, the fluid kinetic energy density, given by $w \gamma^{2} v^{2}$, is concentrated behind the wall, in a region which moves supersonically. For the bag EOS, it is usual to define the efficiency factor $\kappa_{\mathrm{fl}}$ as the fraction of the released vacuum energy which goes into bulk motions of the fluid,

$$
\kappa_{\mathrm{fl}}=\frac{E_{K}}{\Delta \varepsilon V_{b}}=\frac{\rho_{K}}{\Delta \varepsilon}
$$

where $\rho_{K}$ is the average kinetic energy density of the fluid inside the bubble. We shall assume that the bubbles are spherically symmetric and propagate with constant velocity $v_{w}$. In this case, the volume of the bubble is given by $V_{b}=4 \pi\left(v_{w} t\right)^{3} / 3$, and we have

$$
\rho_{K}=\frac{1}{V_{b}} \int_{c_{s} t}^{v_{w} t} 4 \pi r^{2} d r w \gamma^{2} v^{2}
$$

The fluid profiles depend on the variable $\xi=r / t$ and, hence, $\rho_{K}$ does not depend on the time $t$. For spherical bubbles, the functions $w(\xi), v(\xi)$ must be solved numerically. For steady-state walls, $\kappa_{\mathrm{fl}}$ depends only on the parameter $\alpha$ and the wall velocity $v_{w}$ [20, 21, 22], while for runaway walls $\kappa_{\mathrm{fl}}$ depends only on $\alpha$ and $\bar{F}[22$. We shall use the fits for $\kappa_{\mathrm{fl}}$ provided in Ref. [22].

In the runaway case, a fraction of the released vacuum energy goes into kinetic and gradient energy of the wall. In this case we may define an efficiency factor $\kappa_{w}$, in analogy to $\kappa_{\mathrm{f}}$, as $[22]$

$$
\kappa_{w}=\Delta E_{w} /\left(\Delta \varepsilon \Delta V_{b}\right),
$$

where $\Delta E_{w}$ is the energy accumulated in the wall as its volume varies by $\Delta V_{b}$. This coefficient is given by 22 ]

$$
\kappa_{w}=F_{\text {net }}^{U R} / \Delta \varepsilon=\bar{F} / \alpha .
$$

Since the real EOS of the broken-symmetry phase is not of the form (24), in order to utilize Eqs. (25) 34), there is some ambiguity in the definition of the bag parameters $\varepsilon_{b}$ and $a_{b}$. For instance, the vacuum energy density released at the phase transition may be evaluated from the zero-temperature effective potential as $V(0)-V\left(\phi_{m}\right)$. This may be identified with the bag constant $\Delta \varepsilon$, although the minimum $\phi_{m}$ is temperature 
dependent. On the other hand, for the bag EOS we have the relation $L=4 \Delta \varepsilon$. Hence, we could alternatively define the bag constant as $\Delta \varepsilon=L / 4$, with $L=w_{u}\left(T_{c}\right)-w_{b}\left(T_{c}\right)$ computed from the effective potential (4), so that the bag approximation gives the correct value of the latent heat. Notice that for a general model we will have $w_{u}\left(T_{c}\right)-w_{b}\left(T_{c}\right) \neq$ $4\left(V(0)-V\left(\phi_{m}\right)\right)$, and we cannot fit the two quantities with the bag EOS. A third definition is proposed in Refs. [20, 18, namely, $\varepsilon_{b}=\left(\rho_{b}-3 p_{b}\right) / 4$, which is fulfilled for the bag EOS (and, in our case, for the symmetric phase). This gives $\Delta \varepsilon=(\Delta \rho-3 \Delta p) / 4$, with $\Delta \rho=\rho_{u}-\rho_{b}$ and $\Delta p=p_{u}-p_{b}$. For a general model, the quantity $\Delta \varepsilon$ so defined depends on the temperature. At $T=T_{c}$ we have $p_{b}=p_{u}$ and, thus, $\Delta \varepsilon=\Delta \rho / 4=L / 4$, while at $T=0$ we have $\rho_{u, b}=-p_{u, b}$ and, thus, $\Delta \varepsilon=\Delta \rho=V(0)-V\left(\phi_{m}\right)$. Hence, for a phase transition with little supercooling (i.e., $T \simeq T_{c}$ ), defining $\Delta \varepsilon$ in this way will give a bag EOS with the same latent heat as the real model, while for a phase transition with strong supercooling (i.e., $T \ll T_{c}$ ), this definition of $\Delta \varepsilon$ will give the correct value of the vacuum energy density difference.

In order to choose one of these definitions of the bag parameter $\Delta \varepsilon$, we need to consider which of these quantities (the latent heat or the vacuum energy density) is more relevant for the dynamics. The latent heat represents the energy that is released by the phase transition fronts at the critical temperature, while the vacuum energy difference is released at zero temperature. The latent heat reheats the plasma, which affects significantly the wall motion if $T$ is close to $T_{c}$ but not for $T \ll T_{c}$. On the other hand, the difference $V(0)-V\left(\phi_{m}\right)$ gives the net force which drives the wall motion at $T=0$ and is thus relevant in the case of large supercooling. Since we shall consider phase transitions with different amounts of supercooling, it is convenient to adopt the last of the above definitions for the bag constant, namely,

$$
\Delta \varepsilon=(\Delta \rho-3 \Delta p) / 4,
$$

where we shall compute $\Delta \rho=\Delta \mathcal{F}-T d \Delta \mathcal{F} / d T$ and $\Delta p=-\Delta \mathcal{F}$ from Eqs. (115).

Similarly, there is not a unique definition of the effective radiation constant $a_{b}$. Having defined $\varepsilon_{b}$, we may define $a_{b}$ by approximating the remaining part of $\rho_{b}$ by a radiation component, i.e., $a_{b}=\left(\rho_{b}-\varepsilon_{b}\right) / T_{b}^{4}$. On the other hand, for the bag EOS we have the relation $\Delta a T_{c}^{4} / 3=\Delta \varepsilon$. Thus, if we used the definition $\Delta \varepsilon=L / 4$, we might also define $\Delta a=3 L /\left(4 T_{c}^{4}\right)$. Alternatively, we may define the radiation constant from the enthalpy density [20],

$$
a_{b}=3 w_{b} /\left(4 T_{b}^{4}\right) .
$$

At $T=T_{c}$ these definitions coincide. We shall use the latter, with $w_{b}$ computed from (175), since at a given $T$ this definition, together with the one we already adopted for $\varepsilon_{b}$, allow to write the real energy density and pressure in the bag form $p_{b}=-\varepsilon_{b}+a_{b} T_{b}^{4} / 3$, $\rho_{b}=\varepsilon_{b}+a_{b} T_{b}^{4}$ (with $T$-dependent $\varepsilon_{b}$ and $\left.a_{b}\right)$. 


\section{Gravitational wave generation in a first-order phase transition}

\subsection{The mechanisms of GW generation}

The spectrum of gravitational radiation is usually expressed in terms of the energy density per logarithmic frequency $d \rho_{G W} / d \log f$, divided by the critical energy density today $\rho_{c}=$ $3 H_{0}^{2} /(8 \pi G)$, where $H_{0}$ is the Hubble rate today, given by $H_{0}=100 h \mathrm{~km} \mathrm{~s}^{-1} \mathrm{Mpc}^{-1}$, and $h=0.72$. Thus, we have a dimensionless quantity

$$
h^{2} \Omega_{G W}(f)=h^{2} \frac{1}{\rho_{c}} \frac{d \rho_{G W}}{d \log f} .
$$

Several mechanisms of gravitational wave generation have been considered in the literature, namely, bubble collisions, turbulence, and acoustically generated GWs.

The source of gravitational waves are the spatial components of the transverse-traceless part of the stress-energy tensor $T_{i j}$. For a vacuum phase transition, i.e., the case of a scalar field without the hot plasma, the relevant piece is $\partial_{i} \phi \partial_{j} \phi[8]$. Therefore, the source of GWs is concentrated inside the thin regions where $\phi$ varies, i.e., the bubble walls. In this case, it was shown that the system of colliding bubbles can be approximated by a set of overlapping spheres with infinitely thin interfaces [9]. In this "envelop approximation", only the uncollided parts of the spherically symmetric walls are taken into account in the computation of GWs. Thus, the relevant quantity to compute from the scalar field is the integral of $\left(\partial_{r} \phi\right)^{2}$ for a single bubble configuration. In the absence of friction, the walls quickly accelerate to the speed of light, and the kinetic and gradient energies of the bubble wall become equal. Hence, the relevant quantity is the total energy of the wall, which, for a vacuum phase transition is given by the total vacuum energy liberated by the bubble [9], i.e., $E_{w}=4 \pi \int d r r^{2}\left(\partial_{r} \phi\right)^{2}=\Delta \varepsilon V_{b}$, with $V_{b}=4 \pi t^{3} / 3$.

For a thermal phase transition, the bubble walls often reach a terminal velocity due to the friction with the plasma. Generally, this happens in a time which is very short compared to the duration of the phase transition. As a consequence, the walls move with constant velocity, and the energy which is released in the phase transition goes into reheating of the plasma and into bulk fluid motions. These fluid motions also produce gravitational waves. In this case, the relevant part of $T_{i j}$ is $w \gamma^{2} v_{i} v_{j}$. For spherically symmetric walls, this gives the kinetic energy density $w \gamma^{2} v^{2}$. The envelop approximation can still be used [12] if the kinetic energy of the fluid is assumed to be concentrated in a thin region around the wall. The essential difference is that in this case $E_{w}$ is negligible and the result depends on $E_{\mathrm{fl}}=4 \pi \int d r r^{2} w \gamma^{2} v^{2}=\kappa_{\mathrm{fl}} \Delta \varepsilon V_{b}$, where the efficiency factor $\kappa_{\mathrm{fl}}$ is defined in Eq. (31), and $V_{b}=4 \pi v_{w}^{3} t^{3} / 3$.

In the case of a runaway wall, we have $v_{w}=1$ like in the case of a vacuum phase transition. For a runaway wall, an important fraction of the released energy (but not all of it) goes to the bubble wall, i.e., $E_{w}=\kappa_{w} \Delta \varepsilon V_{b}$, with $\kappa_{w}$ defined in Eq. (33). The rest of the vacuum energy goes to reheating and fluid motions, and the relevant quantity for the generation of GWs is the energy in the thin shell around the bubble wall, $E_{w}+E_{\mathrm{fl}}=$ $\left(\kappa_{w}+\kappa_{\mathrm{ff}}\right) \Delta \varepsilon V_{b}$. 
Thus, the general result of the envelop approximation can be written as a function of the ratio

$$
\frac{E_{w}+E_{\mathrm{fl}}}{E_{\mathrm{tot}}}=\frac{\left(\kappa_{w}+\kappa_{\mathrm{ff}}\right) \Delta \varepsilon}{\rho_{\mathrm{tot}}} \equiv \frac{\kappa \Delta \varepsilon}{\rho_{\mathrm{tot}}}
$$

where $\kappa_{w}$ vanishes for $F_{\text {net }}<0$ and is given by (34) for $F_{\text {net }}>0$, and $E_{\text {tot }}=\rho_{\text {tot }} V_{b}$ is the total energy per bubble. As discussed in the previous section, due to energy conservation $\rho_{\text {tot }}$ does not vary significantly during the phase transition, and can be approximated by $\rho_{\text {tot }}=\rho_{u}=a_{u} T_{u}^{4}+\varepsilon_{u}$. In many calculations, the vacuum energy density is assumed to vanish in the broken symmetry phase (which is not necessarily the case at $T \simeq T_{c}$ ). In that case we have $\varepsilon_{u}=\Delta \varepsilon$, and we can write $\rho_{\text {tot }}=a_{u} T_{u}^{4}(1+\alpha)$, which gives

$$
\frac{\kappa \Delta \varepsilon}{\rho_{\text {tot }}}=\frac{\kappa \alpha}{1+\alpha} \text {. }
$$

We shall use instead the expression (38), with $\Delta \varepsilon$ computed from Eq. (35) and $\rho_{\text {tot }}=\rho_{u}$. Nevertheless, we have checked that the approximation (39) does not introduce significant differences in general.

The mechanism described by the envelop approximation is called the "bubble collision" mechanism. In these calculations, the phase transition is simulated by nucleating spherical bubbles at random locations, assuming an exponential nucleation rate $\Gamma(t)=\Gamma_{0} \exp [\beta(t-$ $\left.t_{0}\right)$ ] and a constant wall velocity $v_{w}$. To apply the results of such a calculation to a specific model, $\beta$ can be obtained from

$$
\frac{\beta}{H}=-\frac{T}{\Gamma} \frac{d \Gamma}{d T} \simeq T \frac{d\left(S_{3} / T\right)}{d T}
$$

with $S_{3}$ given by Eq. (9). We shall use the results of Ref. [10] for the spectrum that would be observed today,

$$
h^{2} \Omega_{\mathrm{env}}=1.67 \times 10^{-5}\left(\frac{\kappa \Delta \varepsilon}{\rho_{\mathrm{tot}}}\right)^{2}\left(\frac{100}{g_{*}}\right)^{1 / 3}\left(\frac{H}{\beta}\right)^{2} \frac{0.11 v_{w}^{3}}{0.42+v_{w}^{2}} \frac{3.8\left(f / f_{\mathrm{env}}\right)^{2.8}}{1+2.8\left(f / f_{\mathrm{env}}\right)^{3.8}},
$$

where $f_{\text {env }}$ is the peak frequency today, which is obtained by redshifting the peak frequency $f_{\text {env* }}$ at the moment of the phase transition. The peak frequency is determined by the time scale $\beta^{-1}$. More precisely, it is given by $f_{\text {env* }} \simeq 0.62 /\left(1.8-0.1 v_{w}+v_{w}^{2}\right) \beta$, which gives

$$
f_{\mathrm{env}} \simeq 1.65 \times 10^{-5} \mathrm{~Hz}\left(\frac{g_{*}}{100}\right)^{1 / 6}\left(\frac{T}{100 \mathrm{GeV}}\right)\left(\frac{0.62}{1.8-0.1 v_{w}+v_{w}^{2}}\right) \frac{\beta}{H} .
$$

Notice that the factors of $H$ in these expressions are introduced in order to normalize the constant $\beta$, since one can obtain the ratio $\beta / H$ from the phase transition dynamics. The quantities $g_{*}$ and $T$ appear by assuming that after the phase transition all the vacuum energy is converted into radiation, and thus setting $\rho_{\text {tot }}=\left(\pi^{2} / 30 g_{*}\right) T^{4}$ in the Friedmann equation $H=\sqrt{8 \pi G \rho_{\text {tot }} / 3}$. Hence, the number of degrees of freedom $g_{*}$ corresponds to the broken-symmetry phase and is given by

$$
g_{*}=\left(30 / \pi^{2}\right) a_{b}
$$


which can be obtained from Eq. (36). For strongly first-order phase transitions there can be significant supercooling, which means that the temperature at which bubbles nucleate can be quite smaller than the critical temperature $T_{c} \simeq 100 \mathrm{GeV}$. There will also be some reheating at the end of the phase transition, and the final temperature $T_{\text {reh }}$ after reheating is the one that should be used in Eq. (42). We will estimate this temperature from $\rho_{\text {tot }}=a_{u} T_{p}^{4}+\varepsilon_{u}=a_{b} T_{\text {reh }}^{4}$ (neglecting $\varepsilon_{b}$ ), where $T_{p}$ is the percolation temperature. Nevertheless, in the case of strong supercooling, $T_{\text {reh }}$ will be much closer to $T_{p}$ than to $T_{c}$.

The fluid motions caused by bubble wall motions may remain long after the phase transition is completed. This provides more efficient mechanisms of GW generation. If the fluid motion is turbulent, the eddies can act as a source of GWs after the bubbles have collided. In particular, since the plasma is fully ionized, mhd turbulence can source GWs for several Hubble times. Computing the turbulence spectrum is very difficult. Analytic calculations [16] assuming a characteristic stirring scale $L_{S}$ give a peak frequency $f_{\text {turb* }} \simeq 3.5 / L_{S}$. A fit to these results for the GW spectrum is given in Ref. [54] (see also [55, 27]),

$$
h^{2} \Omega_{\text {turb }}=3.35 \times 10^{-4}\left(\frac{\kappa_{\mathrm{f}} \Delta \varepsilon}{\rho_{\text {tot }}}\right)^{3 / 2}\left(\frac{100}{g_{*}}\right)^{1 / 3} \frac{L_{S} H}{2} \frac{\left(f / f_{\text {turb }}\right)^{3}}{\left(1+f / f_{\text {turb }}\right)^{11 / 3}\left(1+8 \pi f_{*} / H\right)},
$$

where the peak frequency today is given by

$$
f_{\text {turb }}=2.7 \times 10^{-5} \mathrm{~Hz}\left(\frac{g_{*}}{100}\right)^{1 / 6}\left(\frac{T}{100 \mathrm{GeV}}\right)\left(\frac{2}{L_{S} H}\right) .
$$

In the denominator of Eq. (44), $f_{*}$ is the frequency at the time of production. In terms of the redshifted frequency $f$ we may write $f_{*} / H=(3.5 f) /\left(L_{S} H f_{\text {turb }}\right)$. Equations (4445) are usually given in terms of the parameters $v_{w}$ and $\beta$ rather than $L_{S}$. However, we remark that in [16] the spectrum is calculated as a function of the stirring scale $L_{S}$. This scale is then estimated as $L_{S}=2 R_{b} \sim 2 v_{w} \beta^{-1}$, i.e., the typical bubble radius $R_{b}$ is assumed to be given by the wall velocity and the characteristic time scale $\beta^{-1}$. However, as we shall see, for very strong phase transitions the quantity $\beta$ does not give a correct estimation of the time scale. Hence, we prefer to consider a different length scale for the source of turbulence, such as the average distance between bubbles.

Recently, numerical simulations of the field-fluid system were performed [18], finding no indication of fluid turbulence. Nevertheless, it was found that sound waves are a long lasting source of GWs. A simple effective potential was considered, which allows to relate readily its parameters to thermodynamic quantities such as the critical temperature, the latent heat, or the surface tension. However, the number of nucleated bubbles as well as the nucleation temperature $T_{n}$, are set by hand. All the bubbles are nucleated simultaneously and, hence, reach approximately the same size $R_{b}$ at collision. Thus, a free parameter of the simulation is the average distance between bubbles,

$$
d=n_{b}^{-1 / 3}
$$

where $n_{b}$ is the average number density of bubbles. A fit to the resulting spectrum is given in Ref. [27] as a function of the length scale $v_{w} \beta^{-1}$, although $\beta$ is not actually a free parameter in the simulations of Ref. [18]. The dependence on $\beta$ is obtained by estimating 
the average distance as $d=(8 \pi)^{1 / 3} v_{w} / \beta$. This relation results from an analytic estimation of $n_{b}$ for a nucleation rate of the form $\exp (\beta t)$ [36], where $n_{b}$ is evaluated at a time $t$ such that the fraction of volume in the symmetric phase has fallen to $f_{u}=1 / e$. We will instead compute directly the average distance $d$ from Eq. (46), where the number density of bubbles at time $t$ is given by

$$
n_{b}(t)=\int_{t_{c}}^{t} d t^{\prime} \Gamma\left(T^{\prime}\right)\left(\frac{a^{\prime}}{a}\right)^{3}\left(1-f_{b}\right) .
$$

In this equation, the scale factor takes into account the dilution of the number density $\Gamma^{\prime} d t^{\prime}$ and the factor of $f_{u}$ takes into account the fact that bubbles only nucleate in the symmetric phase. Thus we write the GW results from sound waves as

$$
h^{2} \Omega_{s w}=2.65 \times 10^{-6}\left(\frac{\kappa_{\mathrm{fl}} \Delta \varepsilon}{\rho_{\text {tot }}}\right)^{2}\left(\frac{100}{g_{*}}\right)^{1 / 3} \frac{H d}{(8 \pi)^{1 / 3}} \frac{7^{7 / 2}\left(f / f_{s w}\right)^{3}}{\left[4+3\left(f / f_{s w}\right)^{2}\right]^{7 / 2}} .
$$

The peak frequency $f_{s w *}$ is estimated as $f_{s w *}=(2 / \sqrt{3})(8 \pi)^{1 / 3} / d$. After redshifting, we have

$$
f_{s w}=1.9 \times 10^{-5} \mathrm{~Hz}\left(\frac{g_{*}}{100}\right)^{1 / 6}\left(\frac{T}{100 \mathrm{GeV}}\right) \frac{(8 \pi)^{1 / 3}}{H d} .
$$

\subsection{Computation of parameters from phase transition dynamics}

In order to calculate all the quantities appearing in Eqs. (41,49), we shall compute the evolution of the phase transition as explained in Sec. 2, We shall solve numerically the evolution equations (12,14). To compute the nucleation rate, we will solve Eq. (11) iteratively by the overshoot-undershoot method, and then we will integrate Eq. (9). We will track the evolution of the transition through the fraction of volume $f_{b}(t)$. To do that, we will compute the wall velocity from Eq. (30) and then integrate numerically Eqs. (14) and (13).

Notice that the temperature and, hence, all the relevant quantities $v_{w}, \beta, n_{b}$, etc. are constant parameters in the GW equations (41,49). This is due to the simple modeling of the nucleation and growth of bubbles in the corresponding calculations, and is very useful for applications. However, in order to use these results, we need to choose a representative moment in the development of the phase transition. The bubble nucleation becomes appreciable when there is at least one bubble in a Hubble volume $V_{H}=H^{-3}$. This condition is sometimes used to define the nucleation temperature. A rough estimation of this temperature is thus given by the condition $S_{3}(T) / T \lesssim 4 \log (T / H) \simeq 142[30$. However, as the phase transition goes on, the temperature will descend further 6 . For very strong phase transitions this may be important. We thus define the initial time $t_{i}$ by the condition $V_{H} n\left(t_{i}\right)=1$. At $t=t_{i}$ we have $f_{b} \simeq 0$, while the phase transition ends when $f_{b}=1$. Bubbles will effectively begin to meet and collide once their density and size have

\footnotetext{
${ }^{6}$ For the case of very slow bubble walls, there can also be a global reheating during the phase transition. In contrast, for detonations or runaway walls the reheating is localized in a thin shell behind the walls. In this case, temperature homogenization will occur at the end of the phase transition.
} 
become large enough. We shall consider that moment to be the percolation time $t_{p}$, which is given by the condition $f_{b}\left(t_{p}\right) \simeq 0.3[44]^{7}$.

Thus, for using the results of the envelop approximation, we will evaluate the parameters $\beta$ and $v_{w}$ at $t=t_{p}$. For the results on the sound wave mechanism, we will compute the average distance $d$ by evaluating Eqs. (46-47) at $t=t_{p}$. For the turbulence mechanism, there is an ambiguity in the determination of the stirring scale $L_{S}$ at percolation, since there are bubbles of very different sizes. Due to the strong variation of the nucleation rate, smaller bubbles (i.e., those which were nucleated more recently) are much more abundant than larger ones. On the other hand, larger bubbles inject more energy into the turbulence. We shall consider the same scale as in the sound waves mechanism, i.e., $L_{S}=d$. In the next section we discuss further on this ambiguity.

\subsection{Sensitivity curves for eLISA}

As mentioned above, a phase transition at the electroweak scale generally produces a GW stochastic background in the $\mathrm{mHz}$ range, which may therefore be observable at eLISA. The architecture of this space-based observatory is still under debate and several designs are under investigation. Two key design parameters which affect the sensitivity are the arm length and the low-frequency acceleration. The detail about these issues is explained in Ref. [56]. Sensitivity curves for a stochastic background (cosmological sources) are different from the ones calculated for isolated (astrophysical) sources. We will consider sensitivity curves for the designs discussed in Ref. [27]. The data sheet can be found in [57. These designs are generally denoted by N2A5M5L6, N2A1M5L6, N2A2M5L4 and N1A1M2L4. In our graphics we will plot the corresponding sensitivity curves in red, magenta, blue and green, respectively.

\section{Numerical results}

It is well known that one way of achieving a strongly first-order electroweak phase transition is through the addition of bosons with large couplings $h_{i}$ to the Higgs. As already discussed, the one-loop effective potential contains cubic terms $\sim h_{i}^{3} T \phi^{3}$ which strengthen the phase transition by causing a barrier in the free energy. However, such terms are generally suppressed by higher-loop corrections. Nevertheless, if the couplings $h_{i}$ are large enough, the terms $\sim h_{i}^{4} \phi^{4} \log \phi$ in the zero-temperature effective potential (3) may cause a barrier at zero temperature and it is well known that the phase transition may become very strong (see, e.g., [30, 58]). Additional SM singlet scalars also allow new tree-level terms in the scalar potential, which can induce a barrier either at finite temperature or already at zero temperature (even without cubic terms) [41, 59]. These models may give very strong electroweak phase transitions. Alternatively, a non-renormalizable term such as $\phi^{6} / \Lambda^{2}$ can make the phase transition strongly first-order if the scale $\Lambda$ suppressing such operator is in the neighborhood of the electroweak scale [60].

\footnotetext{
${ }^{7}$ Although we are interested in detonations and runaway walls, we shall consider some (fast) deflagration cases for comparison. Since a deflagration wall is preceded by a shock front, in this case we define $t_{p}$ as the time at which "shock bubbles" percolate [4].
} 
One may think that the existence of runaway walls depends directly on the strength of the phase transition. However, as discussed in Ref. [41], if the strength of the transition relies on thermal loops, then the wall will not run away, no matter how strong the phase transition may be. Essentially, this is due to the fact that the net force (20) coincides with the mean field approximation for the free energy difference between the two phases, where the cubic and higher powers of $\phi$ are dropped in the expansion of the thermal part (6) . Moreover, in the example of the SM extension with strongly coupled bosons, in spite of the zero-temperature terms $\phi^{4} \log \phi$, the existence of runaway walls requires fine tuning of the parameters [44]. Runaway solutions are more likely in extensions with tree-level terms.

We wish to investigate the generation of GWs in such strong phase transitions, and in particular to compare models with and without runaway walls. In order to analyze these general features and to keep our results as model independent as possible, we will consider simple models representing the different model classes.

\subsection{The SM with strongly-coupled extra bosons}

Several extensions of the SM consist of adding scalar singlets which are only coupled to the Higgs. We have considered a simple example of such a hidden sector in Ref. [44], consisting of $g$ bosonic degrees of freedom with Higgs-dependent masses of the form $m^{2}=h^{2} \phi^{2}+\mu^{2}$. The phase transition is stronger for higher values of the coupling $h$ and for larger numbers of degrees of freedom, while it becomes weaker for larger $\mu$. Here, we shall consider only the case $\mu=0$. The essential difference of our present treatment with that of [4] (for this particular model) is that we now take into account the saturation of the friction, which leads to higher values of the wall velocity.

For large enough $h$, the effective potential has a barrier at $T=0$, which is reflected in the dependence of the nucleation probability on temperature. This can be appreciated in Fig. 1 (left panel), where we consider the thermal instanton action $S_{3}(T)$ for a few values of $h$ (for $g=2$ ). At $T=T_{c} \sim 100 \mathrm{GeV}$, the instanton action diverges, which means that the nucleation rate vanishes. As $T$ descends below $T_{c}, S_{3}$ decreases and the nucleation rate increases. This is because the minima are no longer degenerate and the barrier between them is smaller. For low values of $h$, the barrier between minima disappears at a certain temperature $T_{0}$. In such a case, $S_{3}$ vanishes at $T=T_{0}$ (see the dashed-dotted line). Therefore, at $T=T_{0}$ the nucleation rate becomes extremely high, $\Gamma \sim T^{4}$, and the phase transition will end before the system reaches this temperature. However, for high enough values of $h$, there is a barrier still at $T=0$. Due to this barrier, the probability of thermal activation will decrease again for small temperatures. Indeed, we see that, for higher values of $h, S_{3} / T$ increases again below $T \sim 50 \mathrm{GeV}$.

As already mentioned, the nucleation becomes effective for $S_{3} / T \approx 142$. Although this is a rough approximation, the qualitative picture is correct. We see, for instance, that for $h=1.82$ the phase transition will occur at $T \approx 90 \mathrm{GeV}$ and for $h=1.88$ it will occur at $T \approx 80 \mathrm{GeV}$, while for values of $h$ close to 1.94 it may happen in the range $40 \mathrm{GeV} \lesssim T \lesssim 60 \mathrm{GeV}$ and for higher values of $h$ it will never happen. The amount of supercooling as a function of $h$ is shown in the central panel of Fig. 1. We see that, indeed, for $h \simeq 1.94$ the nucleation of bubbles begins at a temperature $T \lesssim 50 \mathrm{GeV}$. However, at 

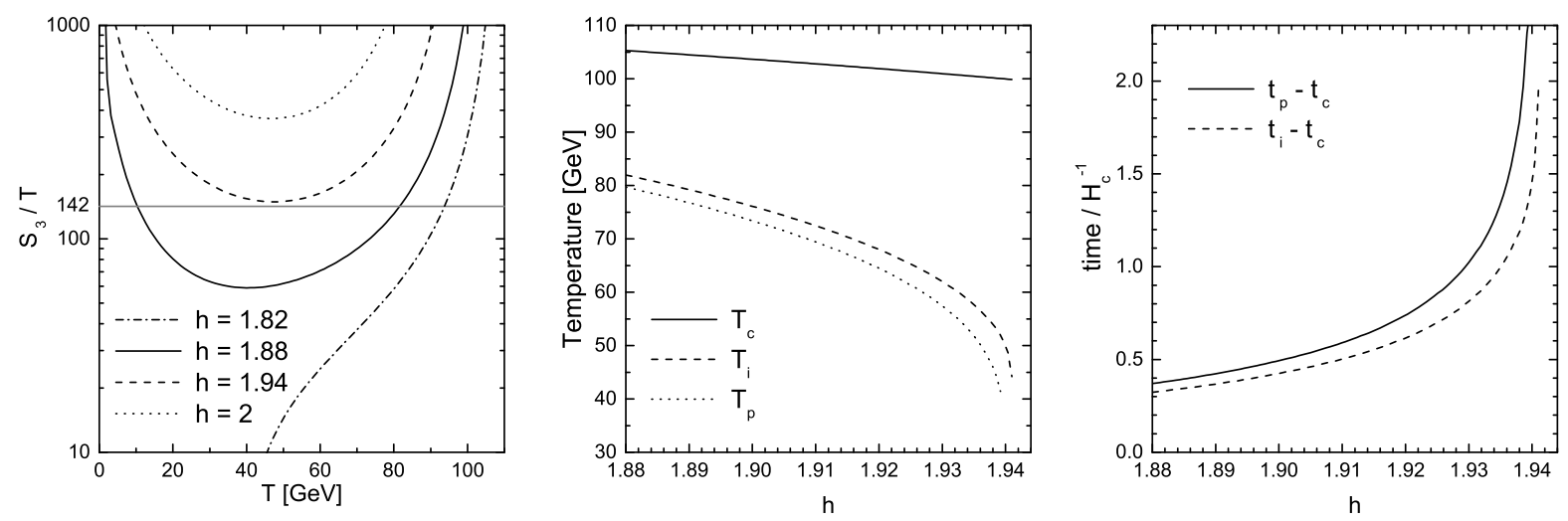

Figure 1: Left: thermal instanton action as a function of temperature, for $g=2$ and several values of $h$. Center: the critical temperature $T_{c}$, the temperature $T_{i}$ at the onset of nucleation, and the temperature $T_{p}$ at percolation. Right: time intervals between the temperature $T_{c}$ and the temperature $T_{i}$ (dashed line) and between $T_{c}$ and $T_{p}$ (solid line).

this point the temperature decreases abruptly as a function of $h$, and there is a critical value $h_{\max }$ for which the phase transition occurs at $T=0$. Beyond this maximum value, the system will remain stuck in the metastable phase, and the phase transition will never end.

In the right panel of Fig. 1 we show the time it takes, once the universe arrives at the critical temperature, to reach the onset of nucleation and then to percolate. We see that for $h>h_{\max }$ the universe remains stuck in the false vacuum and, thus, enters an inflationary era [30, 58]. For GW generation we are interested in very strong phase transitions. However, we are limited by the condition $h<h_{\max }$. Therefore, considering a higher value of $g$ will not change the situation significantly. Indeed, for $g=12$, it was found in Ref. [44] that the maximum value of $h$ is smaller, $h_{\max } \simeq 1.2$. As a consequence, the maximum strength of the phase transition is not higher than for the case of $g=2$. Moreover, the friction is larger and the wall velocity smaller. Here we shall consider only the case $g=2$.

In Fig. 2 we plot the wall velocity for this model, for values of $h$ which give supersonic velocities. In this work we are interested in the detonation and runaway cases. Nevertheless, we show a small part 8 of the deflagration range for comparison. For the deflagration case we used the planar wall approximation (see Ref. 44]). Notice that in this range the deflagrations are supersonic. The wall velocity has a jump from deflagration to detonation solutions at $h \simeq 1.9$. In fact, around this value we have coexistence of a deflagration and a detonation solution. We use the criterion that, in such a case, the detonation is the one that will be realized in the phase transition [61. We see that runaway solutions appear very close to the limiting value $h=h_{\max }$. Therefore, in this model, obtaining runaway walls requires fine tuning of the parameters.

In Fig. 3 we consider the GW production for this model. In the left panel we compare

\footnotetext{
${ }^{8}$ Strongly first-order phase transitions (i.e., with $\phi / T \gtrsim 1$ ) exist already for $h \sim 1.2$. In spite of this, below $h=1.9$ we have deflagration walls. Thus, in comparison, detonation solutions exist in a small range. This is due to the large friction force of these strongly coupled particles.
} 


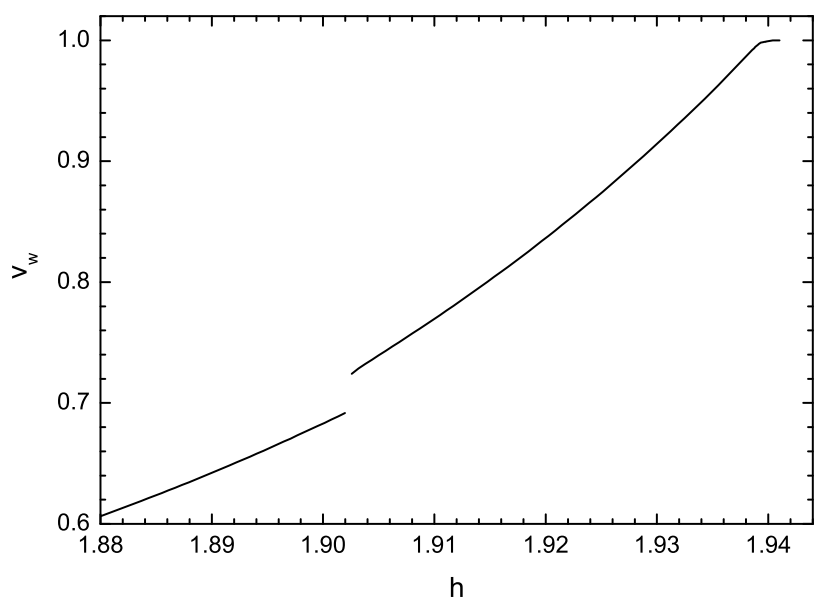

Figure 2: The wall velocity for the SM with $g=2$ extra bosons with mass $m=h \phi$.

the peak amplitude of the GW spectrum for the different formation mechanisms9. Notice that for very strong phase transitions the signal from bubble collisions surpasses the intensity of the other two, and this happens in the detonation regime. However, this result should be considered with caution. Indeed, we find that near $h_{\max }$ the parameter $\beta$ (computed at $t=t_{p}$ ) becomes negative before reaching the value $h_{\max }$. Thus, the growth of the bubble-collisions curve is due in part to the blow-up of $\beta^{-1}$, while the actual duration of the phase transition is still finite. This happens because the supercooling is such that the temperature reaches the minimum of $S_{3} / T$ (we are in the situation of the dashed line in the left panel of Fig. 10. This indicates that the nucleation rate is no longer of the form $\exp (\beta t)$, with constant $\beta$ (i.e., $S_{3} / T$ should be approximated by a quadratic function of $t$ rather than by the linear function $\sim \beta t$ ). Hence, the results (41,42) from the bubble collision simulation break down. As a consequence, we cannot compute the signal from bubble collisions beyond a certain value of the parameter $h$.

In the right panel of Fig. 3 we show the peak intensity vs. the peak frequency for the same range of values of $h$, together with the sensitivity curves for the four eLISA configurations mentioned in Sec. 3. We see that, although the intensity of the signal from bubble collisions grows for very strong phase transitions, its frequency decreases significantly as well (due to the divergence of $\beta^{-1}$ ), departing from the eLISA sensitivity. On the other hand, the signals from turbulence and sound waves have similar frequencies, but the one from sound waves dominates.

The stars on the curves indicate three benchmark points in the range of the parameter $h$. One of them corresponds to the beginning of the detonation range, another one corresponds to a fast detonation, and a third one corresponds to a very strong phase transition, but still with a positive $\beta$. Some of the properties of the phase transition for these benchmark points are displayed in Table 1. In Fig. 4 we plot the spectra from the different sources and the total spectrum (assuming that the corresponding contributions to the stochastic GW background combine linearly [27]).

\footnotetext{
${ }^{9}$ In Ref. [44] we obtained higher amplitudes and smaller frequencies for the GWs from turbulence, since we assumed that the characteristic stirring length was given by the largest bubbles.
} 

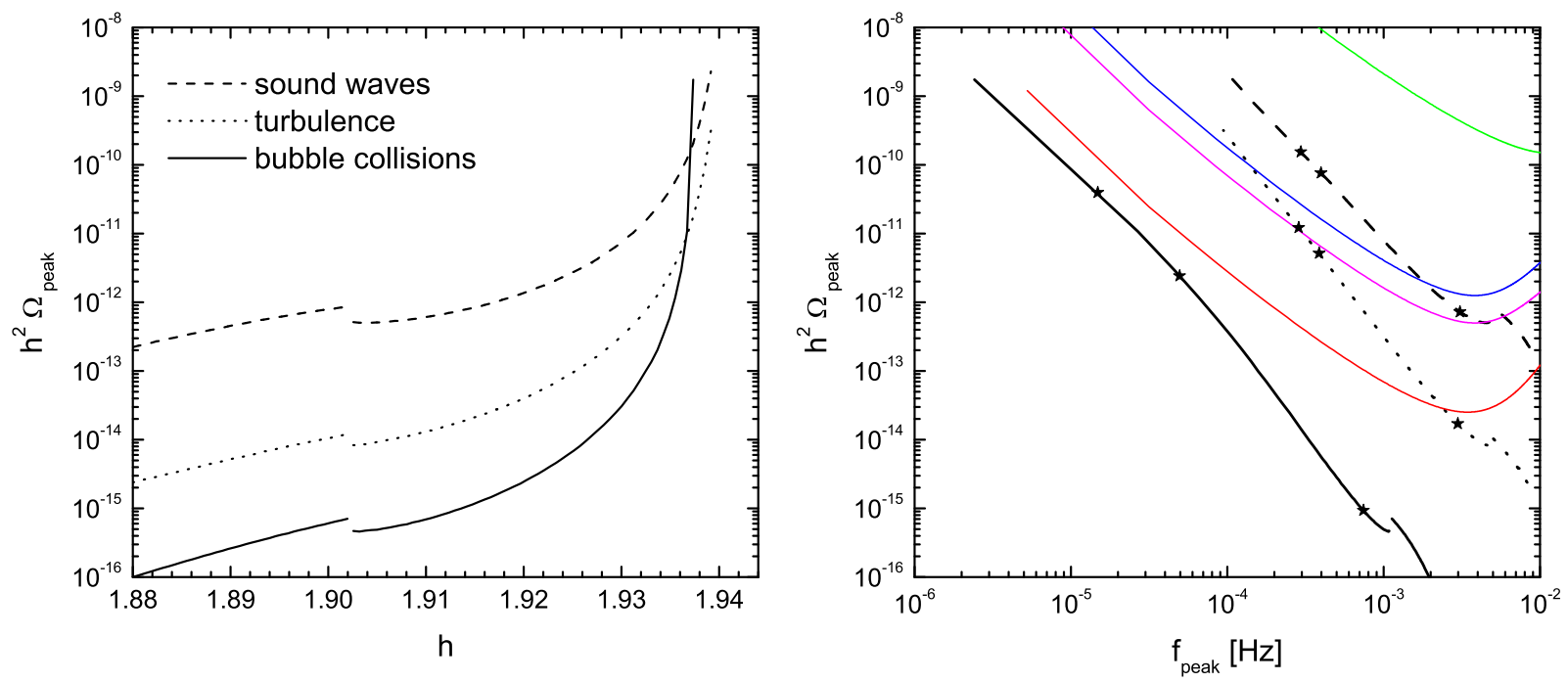

Figure 3: Left panel: peak amplitude of the three components of the GW spectrum for the SM with $g=2$ extra boson d.o.f., as a function of the coupling $h$. Right panel: peak amplitude vs. peak frequency. The stars indicate the values of $h$ given in Table 1. The colored lines correspond to the sensitivity curves of eLISA discussed in Sec. 3 .

\begin{tabular}{|c|c|c|c|c|c|c|c|}
\hline$h$ & $T_{p}[\mathrm{GeV}]$ & $\alpha$ & $\beta / H$ & $H d$ & $v_{w}$ & $g_{b}$ & $\phi / T$ \\
\hline 1.913 & 68.1 & 0.057 & 241.5 & 0.013 & 0.789 & 104 & 3.6 \\
\hline 1.936 & 50.3 & 0.187 & 23.8 & 0.075 & 0.967 & 103 & 4.85 \\
\hline 1.937 & 48.3 & 0.219 & 7.4 & 0.098 & 0.977 & 103 & 5.05 \\
\hline
\end{tabular}

Table 1: Some characteristics of the electroweak phase transition (the temperature at percolation, $T_{p}$, the parameters $\alpha$ and $\beta$, the average distance $d$ between centers of nucleation, the wall velocity, the number of degrees of freedom in the broken-symmetry phase at the end of the phase transition, and the order parameter $\phi / T$ at $T=T_{p}$ ) for a few benchmark values of the coupling $h$ of the extra bosons with the Higgs.

\subsection{The SM with tree-level cubic terms}

In order to investigate the possible effects of tree-level terms, we shall add a cubic term $\propto \phi^{3}$ to the tree-level potential. This extension of the SM must be regarded as a toy model, since such a cubic term cannot be constructed with the Higgs doublet. In this toy model, the field $\phi$ may represent a trajectory in the space of two fields [62]. Adding such a cubic term alone to the potential (11) shifts the tree-level values of the minimum, the Higgs mass, and the true vacuum energy density. We thus add the terms

$$
-A \phi^{3}+\frac{3}{4} A v \phi^{2}+\frac{3}{8} \frac{A}{v} \phi^{4}-\frac{1}{8} A v^{3},
$$

so that we have $V(v)=0, V^{\prime}(v)=0$, and $V^{\prime \prime}(v)=2 \lambda v^{2}$. Since the strength of the phase transition will be dominated by this tree-level modification, we do not need to consider strongly coupled extra particles.

The wall velocity for this model was previously considered in Ref. [52], obtaining 

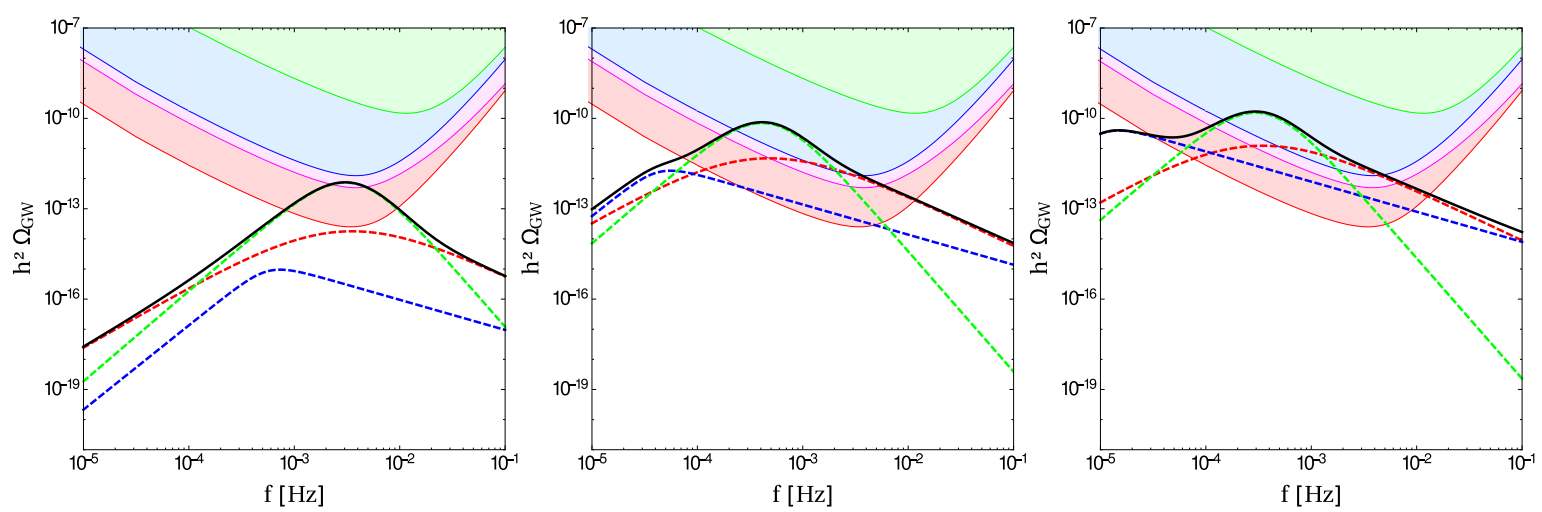

Figure 4: The GW spectra for the benchmark points of Table 1. The blue dashed line denotes the contribution from bubble collisions, the red dashed line the contribution from mhd turbulence, the green dashed line the contribution from sound waves, and the black line the sum of the three signals. The shaded areas represent the regions detectable by the different eLISA configurations.

detonations for $A \gtrsim 20 \mathrm{GeV}$, with wall velocities $v_{w} \lesssim 0.75$. However, the saturation of the friction was not taken into account. In Fig. 5 we plot $v_{w}$ as a function of $A$ (left panel). For $A \lesssim 21 \mathrm{GeV}$ we have subsonic deflagrations, for $21 \mathrm{GeV} \lesssim A \lesssim 32 \mathrm{GeV}$ we have detonations, and for $A \gtrsim 32 \mathrm{GeV}$ we have runaway walls. For $A \simeq 39 \mathrm{GeV}$, the phase transition becomes too strong, like in the previous model. The right panel of Fig. 5 shows the net force normalized to the vacuum energy density, which also gives the efficiency factor $\kappa_{w}$.

In the left panel of Fig. 6 we plot the peak amplitude of the spectrum as a function of the parameter $A$. We see that for detonations the signal from the envelop approximation is smaller than those from turbulence and sound waves, but it begins to grow as soon as runaway solutions appear, and gradually approaches the intensity of the other two signals. In the right panel of Fig. 6] we plot the peak amplitude vs. the peak frequency for the three signals. Although the amplitude $\Omega_{\text {env }}$ blows up as the phase transition becomes very strong, the frequency $f_{\text {env }}$ decreases due to the sudden increase of $\beta^{-1}$, departing from the peak sensitivity of eLISA. The same happened in the previous model. Nevertheless, in this case the signal from bubble collisions is stronger and has a possibility of being observed by the most sensitive eLISA configuration. This is due to the appearance of runaway solutions. For the same reason, the signals from turbulence and sound waves do not reach the intensities they achieved in the previous model.

We consider again some benchmark points on these curves; one of them in the detonation range and the other two in the runaway range. Some characteristics of the phase transition for these points are shown in Table 2, and the corresponding spectra are plotted in Fig. 7. 

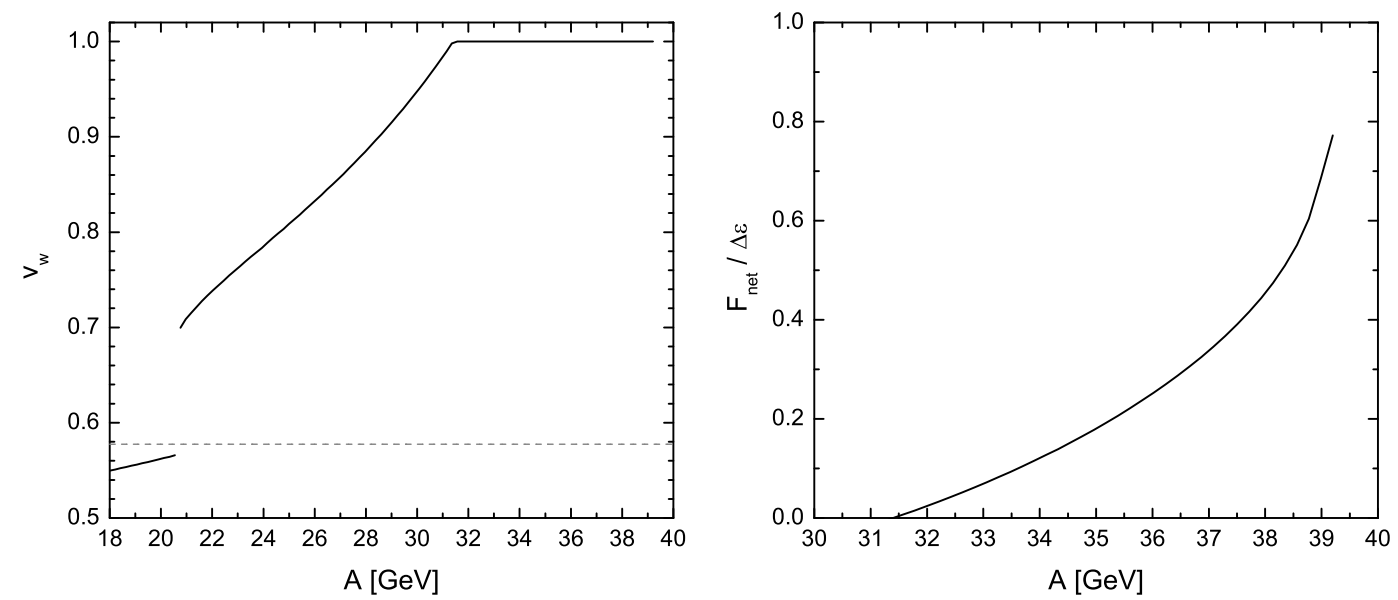

Figure 5: Wall velocity (left panel) and net force (right panel) for the model with a cubic term, as a function of the parameter $A$.

\begin{tabular}{|c|c|c|c|c|c|c|c|}
\hline$A[\mathrm{GeV}]$ & $T_{p}[\mathrm{GeV}]$ & $\alpha$ & $\beta / H$ & $H d$ & $v_{w}$ & $g_{b}$ & $\phi / T$ \\
\hline 30.3 & 90.3 & 0.021 & 886.6 & 0.004 & 0.958 & 103 & 2.5 \\
\hline 36.9 & 65.3 & 0.079 & 214.9 & 0.018 & 1 & 100 & 3.6 \\
\hline 38.7 & 49.8 & 0.237 & 26.4 & 0.074 & 1 & 96.5 & 4.9 \\
\hline
\end{tabular}

Table 2: Some characteristics of the electroweak phase transition (as in Table 1) for three benchmark points of the SM with a term $-A \phi^{3}$.

\subsection{The SM with higher dimensional operators}

We shall now consider an effective theory with a sextic term of the form

$$
\frac{\left(\phi^{2}-v^{2}\right)^{3}}{8 \Lambda^{2}}
$$

which does not shift the tree-level values of the minimum, Higgs mass, and vacuum energy density. For $\Lambda \lesssim 840 \mathrm{GeV}$ the quartic term of the potential becomes negative, which is allowed by the presence of the sextic term. For $\Lambda \lesssim 600 \mathrm{GeV}$ the quadratic term of the zero-temperature potential becomes positive and we have a barrier at zero temperature. We shall not discuss here the possible tension of this kind of model with LHC bounds, and we shall only restrict the value of the cutoff $\Lambda$ by the requirement that the phase transition completes in a few Hubble times, like in the previous cases. This allows values of the cutoff as low as $\Lambda \simeq 550 \mathrm{GeV}$.

We show the wall velocity in the left panel of Fig. 8 and the net force in the right panel. There is a detonation range 10 for $580 \mathrm{GeV} \lesssim \Lambda \lesssim 610 \mathrm{GeV}$, while below $\Lambda \approx 580 \mathrm{GeV}$ we have runaway walls.

In Fig. 9 we show the GW signals. As in the previous case, the existence of runaway solutions enhances the signal from bubble collisions, which gets close to the other signals for very strong phase transitions. On the other hand, all the intensities are higher than

\footnotetext{
${ }^{10}$ This range is relatively small in comparison with the deflagration range. Indeed, for $\Lambda \lesssim 840 \mathrm{GeV}$ we already have $\phi / T>1$, and we have deflagrations from this value to $610 \mathrm{GeV}$.
} 

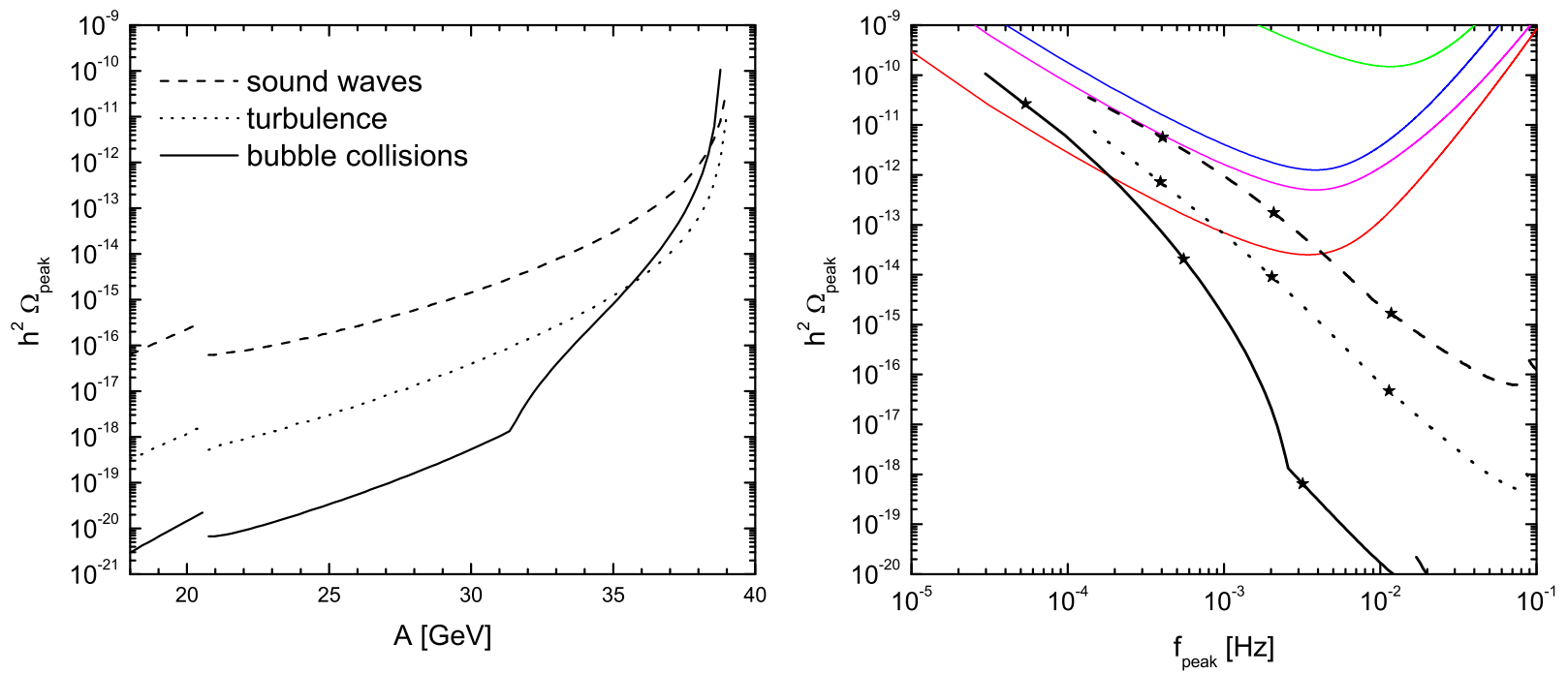

Figure 6: As in Fig. 3, but for the model with a cubic term $-A \phi^{3}$. The stars indicate benchmark points described in Table 2 .
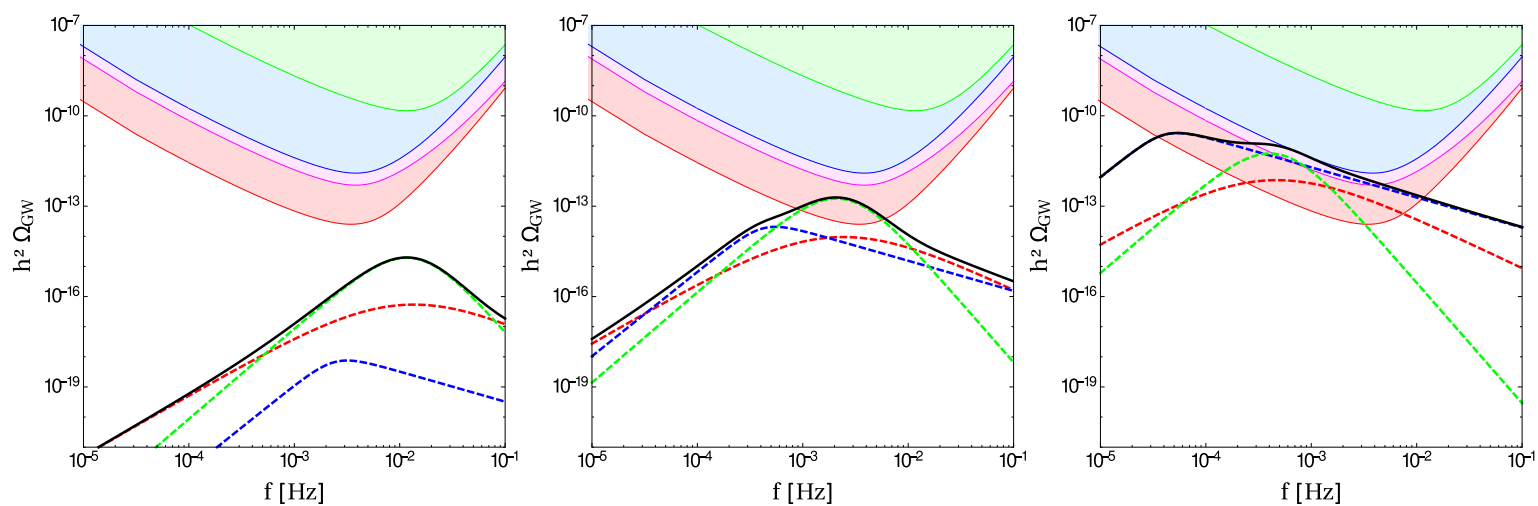

Figure 7: As in Fig. 4, but for the benchmark points of Table 2 for the model with a cubic tree-level term.

for the previous model. We have chosen three benchmark points in the runaway region. The corresponding values of $\Lambda$ and the properties of the phase transition are given in Table 3, and the spectra are plotted in Fig. 10.

\subsection{Discussion}

For all the extensions of the SM considered above, there is a range of the new-physics parameters $h, A$, and $\Lambda$ for which the phase transition has detonations or runaway walls. We observe that models with both kinds of solutions may give a strong signal of gravitational waves. However, as expected, the existence of runaway solutions favors the signal from bubble collisions, while the existence of steady-state walls favors the signals from turbulence and sound waves. Nevertheless, even in the runaway range, the intensity of the signals from fluid motions grows with the strength of the transition. In particular, the signal from sound waves generally dominates in the sensitivity range of eLISA. More- 

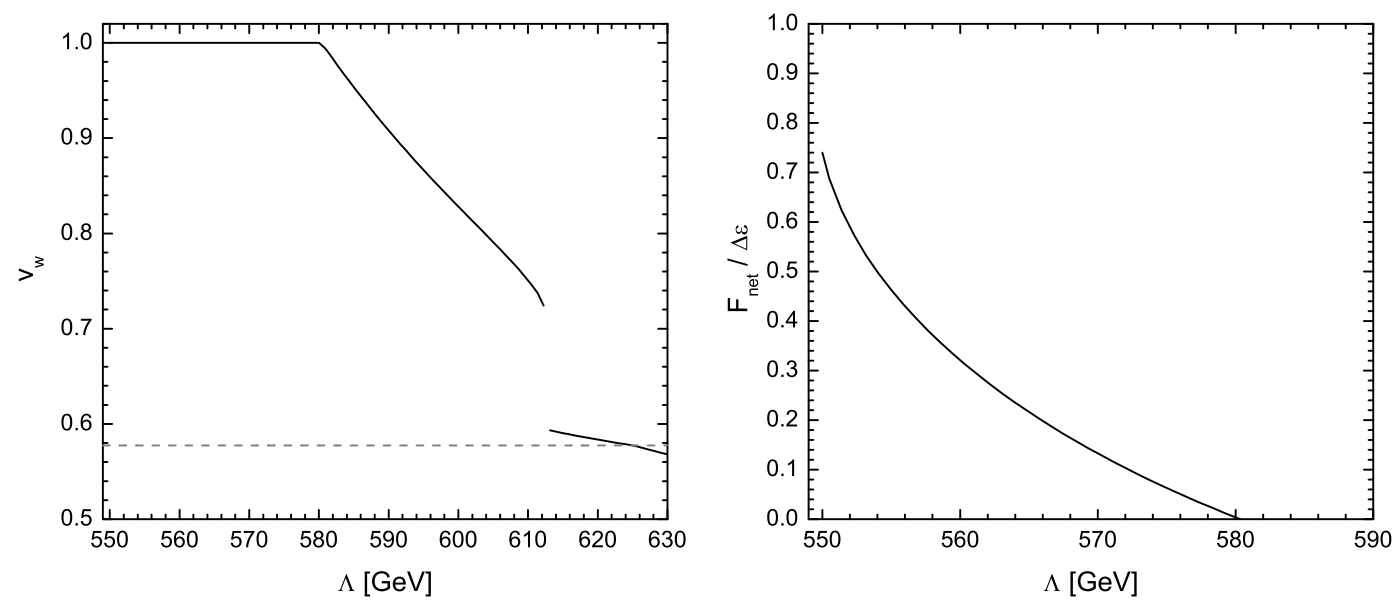

Figure 8: The wall velocity (left panel) and net force (right panel) for the SM with a sextic operator, as functions of the cutoff $\Lambda$.

\begin{tabular}{|c|c|c|c|c|c|c|c|}
\hline$\Lambda[\mathrm{GeV}]$ & $T_{p}[\mathrm{GeV}]$ & $\alpha$ & $\beta / H$ & $H d$ & $v_{w}$ & $g_{b}$ & $\phi / T$ \\
\hline 565.5 & 57.5 & 0.09 & 379 & 0.01 & 1 & 98.5 & 4.2 \\
\hline 553.5 & 43 & 0.28 & 122 & 0.03 & 1 & 94.5 & 5.7 \\
\hline 550.5 & 34.5 & 0.68 & 27.6 & 0.086 & 1 & 91 & 7.1 \\
\hline
\end{tabular}

Table 3: Some characteristics of the phase transition (as in Table 1) for three benchmark points of the model with a dimension six operator with a low cutoff $\Lambda$.

over, it seems that only for phase transitions with runaway walls the signal from bubble collisions has a chance of being observable.

Since the dominant mechanism is suppressed for runaway walls, the main effect of the latter is to weaken the GW signal. As a consequence, the SM extension with extra bosons gives the strongest signal. In Fig. 11 we compare the results from the sound waves mechanism for the different models.

The case of strongly coupled extra bosons clearly deserves further investigation. Here, we have only considered a mass of the simple form $m(\phi)=h \phi$ and the particular case of $g=2$ d.o.f. For a more general mass of the form $m^{2}=h^{2} \phi^{2}+\mu^{2}$, the phase transition is generally weaker. On the other hand, for a larger number of degrees of freedom, the phase transition is stronger but the friction is larger, which prevents higher wall velocities. In particular, for $g=12$ we obtain deflagrations in the whole range of $h$ (up to $h_{\max }$ ). Nevertheless, as can be seen in Fig. 11, for this kind of model deflagrations may generate an important GW signal. As already mentioned, deflagrations reheat the plasma outside the bubbles. This makes the computation of the wall velocity more difficult, and also complicates the treatment of bubble nucleation 11 . Therefore, the general treatment of a phase transition mediated by deflagrations should be improved before embarking in a complete exploration of the parameter space. We shall address this problem elsewhere.

We remark that the computation of gravitational waves from phase transitions is

\footnotetext{
${ }^{11}$ To compute the wall velocity in this case we used the planar wall approximation and we stopped the evolution of the phase transition at the percolation of shock bubbles (see 44 for details).
} 

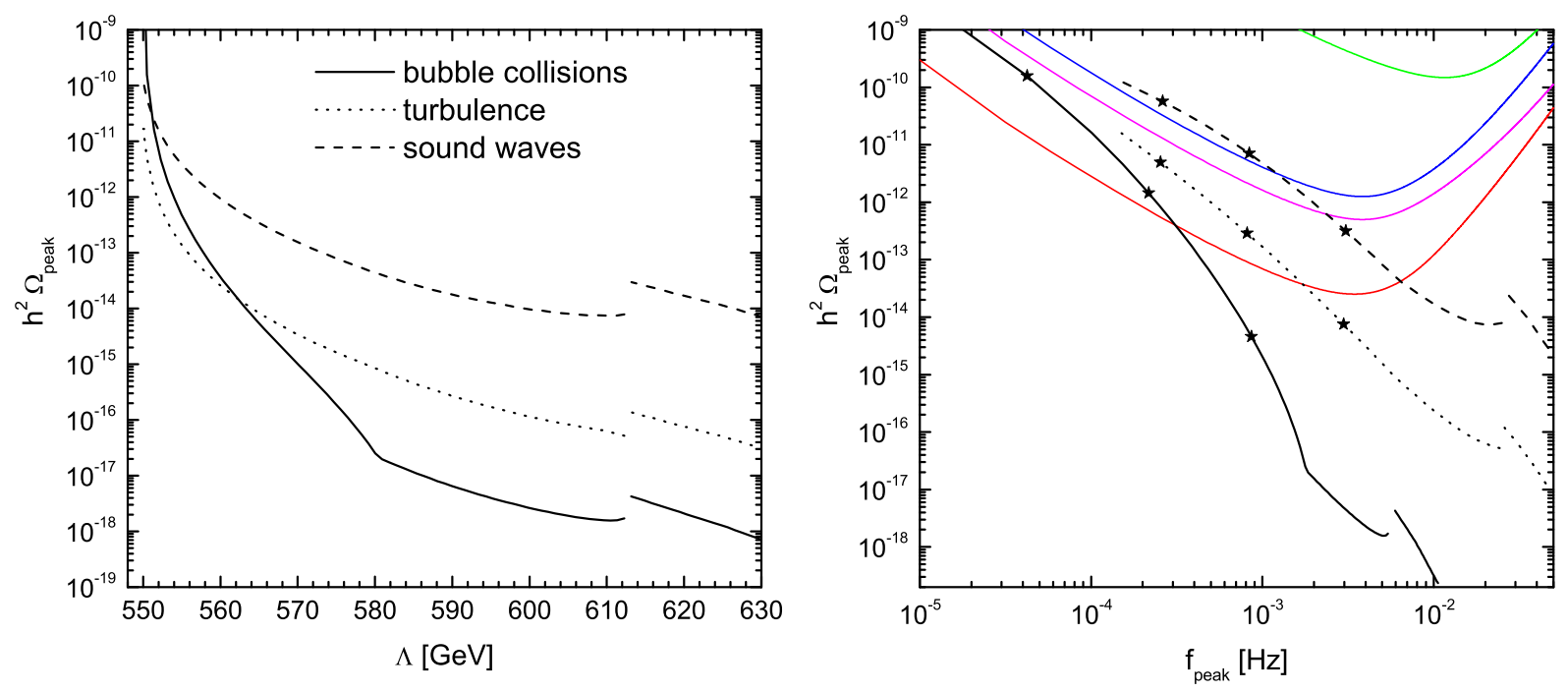

Figure 9: As in Fig. 3, but for the model with a sextic term $\phi^{6} / \Lambda$. The stars indicate benchmark points described in Table 3 .
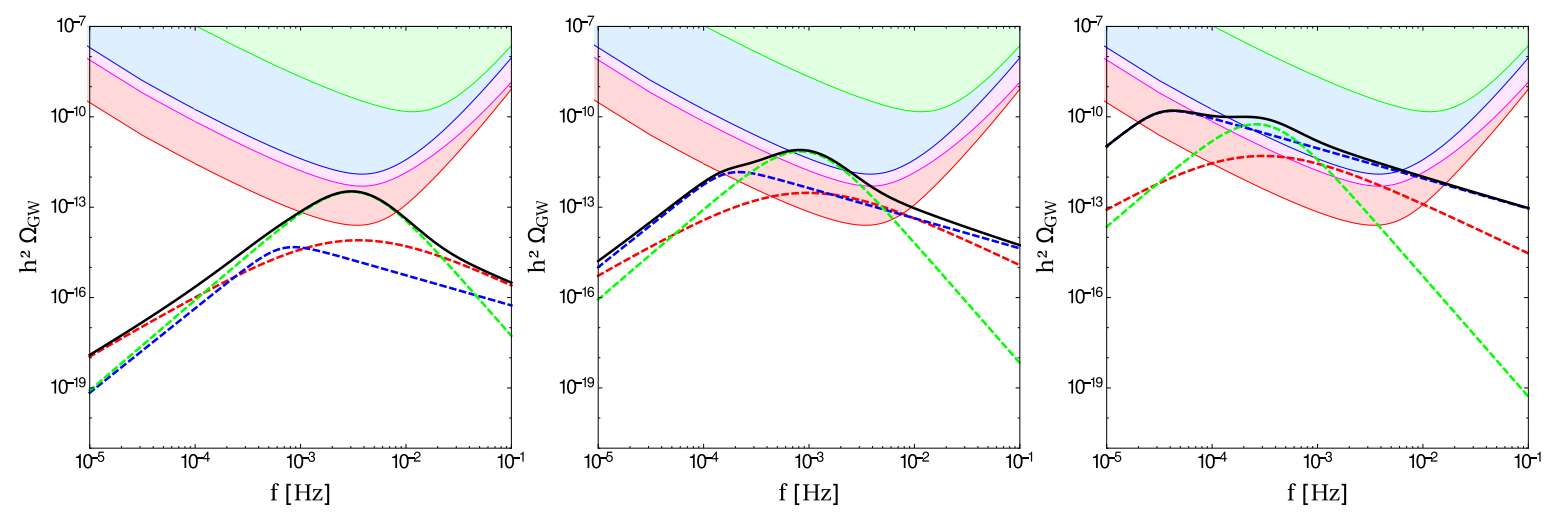

Figure 10: As in Fig. 4, but for the benchmark points of Table 3 for the model with a sextic term.

generally affected by ambiguities in the determination of some quantities. One important source of uncertainty in the cases of turbulence and sound waves is the assumption of a single length scale (either by assuming a single stirring scale or by nucleating all bubbles simultaneously). In a real phase transition there are different length scales (the sizes of bubbles), and we had to choose a characteristic size in order to use those results. In the case of bubble collisions, as already discussed, the approximation $\Gamma \propto \exp (\beta t)$ breaks down for very strong supercooling. This introduces an uncertainty in the characteristic time scale $\beta^{-1}$.

In order to assess the impact of these uncertainties, we compare in Fig. 12 three different scales which are often used in the literature. Besides the average bubble distance $d=n_{b}^{-1 / 3}$ used in this work, we consider the estimation $2 v_{w} \beta^{-1}$ for the bubble diameter, and the diameter $2 R_{b}\left(t_{i}, t_{p}\right)$ of the "largest bubbles", i.e., those which expanded since the onset of nucleation $t_{i}$ until the percolation time $t_{p}$. The plot corresponds to the case of the SM with a cubic term; a similar plot for the case of extra bosons can be found in 


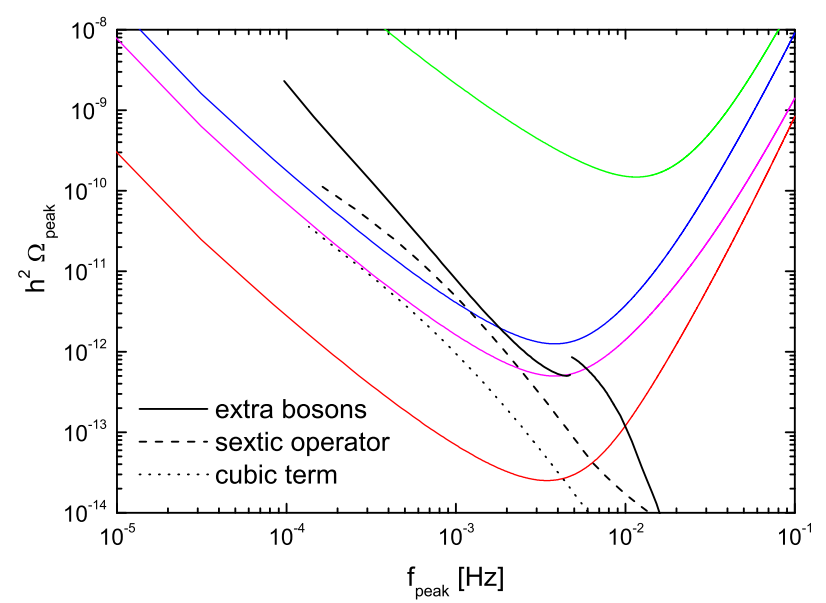

Figure 11: The peak amplitude vs. the peak frequency corresponding to the sound waves mechanism, for the three extensions of the SM considered in this work.

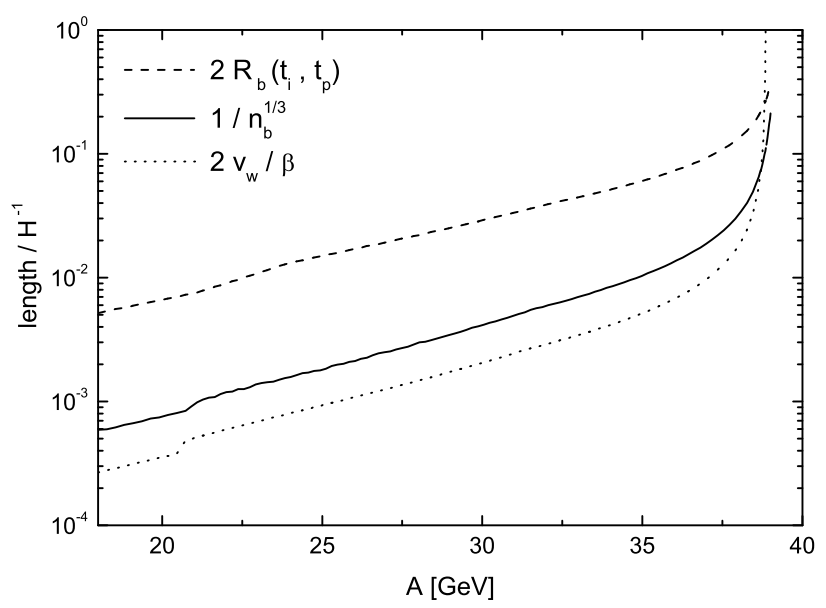

Figure 12: Different length scales present in the phase transition, for the extension of the SM with a tree level term.

Ref. [44]. We see that the average bubble separation is generally an order of magnitude smaller than the scale of the largest bubbles, and the scale $v_{w} \beta^{-1}$ is even smaller. As shown in Ref. 44], we have the relation $R_{b}\left(t_{i}, t_{p}\right) \sim 3 \log (\beta / H) v_{w} \beta^{-1}$. Had we used the scale $R_{b}$ instead of $d$, we would have obtained GWs of quite higher intensity but with smaller frequencies. On the other hand, for very strong phase transitions the distance $d$ approaches $R_{b}$, while $v_{w} \beta^{-1}$ diverges, as already discussed.

\section{Conclusions}

We have discussed the generation of gravitational waves in very strong electroweak phase transitions, such that fast detonations or runaway walls are possible. For that aim we have studied extensions of the SM with extra bosons, with tree-level terms, and with nonrenormalizable operators. By considering simple examples of these three classes of models, 
we focused on the dynamics of the phase transition. Specifically, we have considered model parameters which give phase transitions with considerable amounts of supercooling. We have explored the model parameters up to the limit in which the universe remains stuck in the metastable vacuum and the phase transition does not come to an end.

In contrast to earlier studies, we aimed to consider the dynamics of the phase transition in the most realistic manner possible. In the first place, we pointed out the uncertainties and ambiguities in the calculations of the quantities $\alpha, \beta$, and other parameters which are relevant for GW generation, and we discussed the best way to estimate these parameters. In the second place, we computed the development of the phase transition from the onset of nucleation to percolation. The calculation included the evaluation of model-dependent friction parameters which determine the wall velocity. In this regard, we would like to comment that in recent papers the computation of the wall velocity was avoided by either considering only models which give runaway walls [33], fixing by hand the value of the detonation velocity [27], or assuming a Jouguet detonation [32].

We have compared the gravitational wave spectra generated via the three known mechanisms, namely, bubble collisions, fluid turbulence and sound waves, and we have discussed the possibility of observing these signals at the eLISA interferometer. For runaway walls, part of the energy goes to the fluid and part goes to the bubble wall. In comparison with the stationary motion, this strengthens the signal from bubble collisions and weakens the signals from turbulence and sound waves. In spite of this, we have found that the latter two are stronger in most of the parameter regions. This is because the fluid motions are long-lasting sources of GWs. In any case, the signal from bubble collisions increases significantly for very strong phase transitions. This is in part due to the presence of runaway walls, but also due to the vanishing of the parameter $\beta$, and deserves further investigation. Nevertheless, in the extreme cases in which the amplitude from bubble collisions surpasses those from the other sources, its peak frequency decreases significantly as well. As a consequence, this signal is never dominant in the region which is detectable by eLISA.

Our main conclusion is that, since the GW signals from sound waves and turbulence are generally the dominant ones, detonations are preferable over runaway walls for phase transitions of similar strength. As a consequence, among the models we considered, the SM extension with strongly-coupled extra bosons has the best prospects for being detected at eLISA.

\section{Acknowledgements}

This work was supported by Universidad Nacional de Mar del Plata, Argentina, grant EXA699/14, and by FONCyT grant PICT 2013 No. 2786. The work of L.L. was supported by a CONICET fellowship.

\section{References}

[1] M. Maggiore, Phys. Rept. 331, 283 (2000) gr-qc/9909001. 
[2] N. Seto, S. Kawamura and T. Nakamura, Phys. Rev. Lett. 87, 221103 (2001) astro-ph/0108011; S. Kawamura, T. Nakamura, M. Ando, N. Seto, K. Tsubono, K. Numata, R. Takahashi and S. Nagano et al., Class. Quant. Grav. 23, S125 (2006); S. Kawamura et.al., J. Phys.: Conf. Ser. 122, 012006 (2008).

[3] V. Corbin and N. J. Cornish, Class. Quant. Grav. 23 (2006) 2435 [gr-qc/0512039]; C. Ungarelli, P. Corasaniti, R. A. Mercer and A. Vecchio, Class. Quant. Grav. 22, S955 (2005) astro-ph/0504294]; C. Cutler and J. Harms, Phys. Rev. D 73, 042001 (2006) gr-qc/0511092]; G. M. Harry, P. Fritschel, D. A. Shaddock, W. Folkner and E. S. Phinney, Class. Quant. Grav. 23, 4887 (2006) [Erratum-ibid. 23, 7361 (2006)].

[4] K. Danzmann and A. Rudiger, Class. Quant. Grav. 20, S1 (2003).

[5] P. Amaro-Seoane et al., GW Notes 6, 4 (2013) arXiv:1201.3621 [astro-ph.CO]]; P. Amaro-Seoane et al., Class. Quant. Grav. 29, 124016 (2012) arXiv:1202.0839 [grqc]]; P. A. Seoane et al. [eLISA Collaboration], arXiv:1305.5720 [astro-ph.CO].

[6] For reviews, see A. G. Cohen, D. B. Kaplan and A. E. Nelson, Ann. Rev. Nucl. Part. Sci. 43, 27 (1993) arXiv:hep-ph/9302210; A. Riotto and M. Trodden, Ann. Rev. Nucl. Part. Sci. 49, 35 (1999) [arXiv:hep-ph/9901362].

[7] M. S. Turner and F. Wilczek, Phys. Rev. Lett. 65, 3080 (1990);

[8] A. Kosowsky, M. S. Turner and R. Watkins, Phys. Rev. Lett. 69, 2026 (1992); Phys. Rev. D 45, 4514 (1992).

[9] A. Kosowsky and M. S. Turner, Phys. Rev. D 47, 4372 (1993).

[10] S. J. Huber and T. Konstandin, JCAP 0809, 022 (2008) arXiv:0806.1828 [hep-ph]].

[11] C. Caprini, R. Durrer and G. Servant, Phys. Rev. D 77, 124015 (2008) arXiv:0711.2593 [astro-ph]].

[12] M. Kamionkowski, A. Kosowsky and M. S. Turner, Phys. Rev. D 49, 2837 (1994).

[13] A. D. Dolgov, D. Grasso and A. Nicolis, Phys. Rev. D 66, 103505 (2002).

[14] G. Gogoberidze, T. Kahniashvili and A. Kosowsky, Phys. Rev. D 76, 083002 (2007).

[15] T. Kahniashvili, G. Gogoberidze and B. Ratra, arXiv:0802.3524 [astro-ph].

[16] C. Caprini, R. Durrer and G. Servant, JCAP 0912, 024 (2009) arXiv:0909.0622 [astro-ph.CO]].

[17] L. Kisslinger and T. Kahniashvili, Phys. Rev. D 92, no. 4, 043006 (2015) doi:10.1103/PhysRevD.92.043006 [arXiv:1505.03680 [astro-ph.CO]].

[18] M. Hindmarsh, S. J. Huber, K. Rummukainen and D. J. Weir, Phys. Rev. Lett. 112, 041301 (2014) arXiv:1304.2433 [hep-ph]]; M. Hindmarsh, S. J. Huber, K. Rummukainen and D. J. Weir, arXiv:1504.03291 [astro-ph.CO]. 
[19] A. Mégevand, Phys. Rev. D 78 (2008) 084003 [arXiv:0804.0391 [astro-ph]].

[20] J. R. Espinosa, T. Konstandin, J. M. No and G. Servant, JCAP 1006, 028 (2010) arXiv:1004.4187 [hep-ph]];

[21] L. Leitao and A. Mégevand, Nucl. Phys. B 844, 450 (2011) arXiv:1010.2134 [astroph.CO]].

[22] L. Leitao and A. Megevand, Nucl. Phys. B 905, 45 (2016) arXiv:1510.07747 [astroph.CO]].

[23] A. Nicolis, Class. Quant. Grav. 21, L27 (2004).

[24] C. Grojean and G. Servant, Phys. Rev. D 75, 043507 (2007).

[25] T. Kahniashvili, A. Kosowsky, G. Gogoberidze and Y. Maravin, Phys. Rev. D 78, 043003 (2008) [arXiv:0806.0293 [astro-ph]].

[26] P. Schwaller, Phys. Rev. Lett. 115, no. 18, 181101 (2015) doi:10.1103/PhysRevLett.115.181101 arXiv:1504.07263 [hep-ph]].

[27] C. Caprini et al., JCAP 1604, no. 04, 001 (2016) [arXiv:1512.06239 [astro-ph.CO]].

[28] R. Apreda, M. Maggiore, A. Nicolis and A. Riotto, Nucl. Phys. B 631, 342 (2002).

[29] S. J. Huber and T. Konstandin, JCAP 0805, 017 (2008) arXiv:0709.2091 [hep-ph]].

[30] J. R. Espinosa, T. Konstandin, J. M. No and M. Quiros, Phys. Rev. D 78, 123528 (2008) [arXiv:0809.3215 [hep-ph]].

[31] J. Kehayias and S. Profumo, JCAP 1003, 003 (2010) [arXiv:0911.0687 [hep-ph]].

[32] M. Kakizaki, S. Kanemura and T. Matsui, Phys. Rev. D 92, no. 11, 115007 (2015) arXiv:1509.08394 [hep-ph]].

[33] S. J. Huber, T. Konstandin, G. Nardini and I. Rues, JCAP 1603, no. 03, 036 (2016) arXiv:1512.06357 [hep-ph]].

[34] B. H. Liu, L. D. McLerran and N. Turok, Phys. Rev. D 46, 2668 (1992); N. Turok, Phys. Rev. Lett. 68, 1803 (1992); M. Dine, R. G. Leigh, P. Y. Huet, A. D. Linde and D. A. Linde, Phys. Rev. D 46, 550 (1992) arXiv:hep-ph/9203203]; S. Y. Khlebnikov, Phys. Rev. D 46, 3223 (1992); P. Arnold, Phys. Rev. D 48, 1539 (1993) arXiv:hep-ph/9302258.

[35] M. Gyulassy, K. Kajantie, H. Kurki-Suonio and L. D. McLerran, Nucl. Phys. B 237, 477 (1984); H. Kurki-Suonio, Nucl. Phys. B 255, 231 (1985); K. Kajantie and H. Kurki-Suonio, Phys. Rev. D 34, 1719 (1986); A. F. Heckler, Phys. Rev. D 51 405, (1995) [arXiv:astro-ph/9407064].

[36] K. Enqvist, J. Ignatius, K. Kajantie and K. Rummukainen, Phys. Rev. D 45, 3415 (1992); 
[37] A. Mégevand and A. D. Sánchez, Nucl. Phys. B 820, 47 (2009) arXiv:0904.1753 [hep-ph]].

[38] G. D. Moore and T. Prokopec, Phys. Rev. D 52, 7182 (1995) arXiv:hep-ph/9506475; Phys. Rev. Lett. 75, 777 (1995) arXiv:hep-ph/9503296.

[39] P. John and M. G. Schmidt, Nucl. Phys. B 598, 291 (2001) [Erratum-ibid. B 648, 449 (2003)];

[40] J. Kozaczuk, JHEP 1510, $135 \quad$ (2015) doi:10.1007/JHEP10(2015)135 arXiv:1506.04741 [hep-ph]].

[41] D. Bodeker and G. D. Moore, JCAP 0905, 009 (2009) [arXiv:0903.4099 [hep-ph]].

[42] T. Konstandin, G. Nardini and I. Rues, JCAP 1409, no. 09, 028 (2014).

[43] M. Laine, Phys. Rev. D 49, 3847 (1994) arXiv:hep-ph/9309242].

[44] L. Leitao, A. Megevand and A. D. Sanchez, JCAP 1210, 024 (2012) arXiv:1205.3070 [astro-ph.CO]].

[45] A. Megevand, JCAP 1307, 045 (2013) [arXiv:1303.4233 [astro-ph.CO]].

[46] W. Buchmuller and O. Philipsen, Nucl. Phys. B 443, 47 (1995) [hep-ph/9411334]; K. Kajantie, M. Laine, K. Rummukainen and M. E. Shaposhnikov, Phys. Rev. Lett. 77, 2887 (1996) [hep-ph/9605288].

[47] M. Quiros, arXiv:hep-ph/9901312.

[48] C. Wainwright, S. Profumo and M. J. Ramsey-Musolf, Phys. Rev. D 84, 023521 (2011) arXiv:1104.5487 [hep-ph]]; C. L. Wainwright, S. Profumo and M. J. RamseyMusolf, arXiv:1204.5464 [hep-ph].

[49] I. Affleck, Phys. Rev. Lett. 46, 388 (1981); A. D. Linde, Nucl. Phys. B 216, 421 (1983) [Erratum-ibid. B 223, 544 (1983)]; Phys. Lett. B 100, 37 (1981).

[50] A. H. Guth and E. J. Weinberg, Phys. Rev. D 23, 876 (1981).

[51] A. Mégevand, Phys. Rev. D 69, 103521 (2004) arXiv:hep-ph/0312305.

[52] A. Megevand and A. D. Sanchez, Nucl. Phys. B 825, 151 (2010) arXiv:0908.3663 [hep-ph]].

[53] G. D. Moore, JHEP 0003, 006 (2000) arXiv:hep-ph/0001274.

[54] C. Caprini, R. Durrer and X. Siemens, Phys. Rev. D 82, 063511 (2010) arXiv:1007.1218 [astro-ph.CO]].

[55] P. Binetruy, A. Bohe, C. Caprini and J. -F. Dufaux, arXiv:1201.0983 [gr-qc].

[56] A. Klein et al., arXiv:1511.05581 [gr-qc]. 
[57] Data sheet by A. Petiteau, http://www.apc.univ-paris7.fr/Downloads/lisa/eLISA/Sensitivity/Cfgv1/StochBkgd/

[58] A. Mégevand and A. D. Sánchez, Phys. Rev. D 77, 063519 (2008) arXiv:0712.1031 [hep-ph]].

[59] J. Choi and R. R. Volkas, Phys. Lett. B 317, 385 (1993) arXiv:hep-ph/9308234; S. W. Ham, Y. S. Jeong and S. K. Oh, J. Phys. G 31, 857 (2005) arXiv:hep-ph/0411352.

[60] C. Delaunay, C. Grojean and J. D. Wells, JHEP 0804, 029 (2008) arXiv:0711.2511 [hep-ph]].

[61] A. Megevand and A. D. Sanchez, Nucl. Phys. B 865, 217 (2012) arXiv:1206.2339 [astro-ph.CO]].

[62] D. J. H. Chung, A. J. Long and L. T. Wang, Phys. Rev. D 87, 023509 (2013). 